\title{
A Class of Approximate Damped Oscillatory Solutions to Compound KdV-Burgers-Type Equation with Nonlinear Terms of Any Order: Preliminary Results
}

\author{
Yan Zhao' and Weiguo Zhang ${ }^{2}$ \\ ${ }^{1}$ College of Engineering, Peking University, Beijing 100871, China \\ ${ }^{2}$ College of Science, University of Shanghai for Science and Technology, Shanghai 200093, China \\ Correspondence should be addressed to Yan Zhao; zhaoyanem@163.com
}

Received 2 June 2014; Accepted 25 August 2014; Published 23 November 2014

Academic Editor: Keshlan S. Govinder

Copyright ( $) 2014$ Y. Zhao and W. Zhang. This is an open access article distributed under the Creative Commons Attribution License, which permits unrestricted use, distribution, and reproduction in any medium, provided the original work is properly cited.

This paper is focused on studying approximate damped oscillatory solutions of the compound KdV-Burgers-type equation with nonlinear terms of any order. By the theory and method of planar dynamical systems, existence conditions and number of bounded traveling wave solutions including damped oscillatory solutions are obtained. Utilizing the undetermined coefficients method, the approximate solutions of damped oscillatory solutions traveling to the left are presented. Error estimates of these approximate solutions are given by the thought of homogeneous principle. The results indicate that errors between implicit exact damped oscillatory solutions and approximate damped oscillatory solutions are infinitesimal decreasing in the exponential form.

\section{Introduction}

The compound KdV-type equation with nonlinear terms of any order

$$
\begin{array}{r}
u_{t}+a u^{p} u_{x}+b u^{2 p} u_{x}+\beta u_{x x x}=0, \\
a, b \in R, \beta>0, p \in N^{+}
\end{array}
$$

is an important model equation in quantum field theory, plasma physics, and solid state physics [1]. In recent years, many physicists and mathematicians have paid much attention to this equation. For example, Wadati $[2,3]$ studied soliton, conservation laws, Bäclund transformation, and other properties of (1) with $p=1$. Dey [1,4] and Coffey [5] obtained the kink profile solitary wave solutions of (1) under particular parameter values and compared them with the solutions of relativistic field theories. In addition, they evaluated exact Hamiltonian density and gave conservation laws. Employing the bifurcation theory of planar dynamical systems to analyze the planar dynamical system corresponding to (1), Tang et al. [6] presented bifurcations of phase portraits and obtained the existence conditions and number of solitary wave solutions. On the assumption that the integral constant $g$ is equal to zero, they obtained some explicit bell profile solitary wave solutions. Liu and Li [7] also studied (1) by the bifurcation theory of planar dynamical systems. In addition to obtaining the same bell profile solitary wave solutions as those given by Tang et al. [6], Liu and Li [7] also presented some explicit kink profile solitary wave solutions. In [8], Zhang et al. used proper transformation to degrade the order of nonlinear terms of (1). And then, by the undetermined coefficients method, they obtained some explicit exact solitary wave solutions. Indeed, the solutions obtained in [6-8] are equivalent under certain conditions.

Dissipation effect is inevitable in practical problem. It would rise when wave comes across the damping in the movement. Whitham [9] pointed out that one of basic problems needed to be concerned for nonlinear evolution equations 
was how dissipation affects nonlinear systems. Therefore, it is meaningful to study the compound KdV-Burgers-type equation with nonlinear terms of any order given by

$$
\begin{array}{r}
u_{t}+a u^{p} u_{x}+b u^{2 p} u_{x}+r u_{x x}+\beta u_{x x x}=0, \\
a, b \in R, \beta>0, r<0, p \in N^{+} .
\end{array}
$$

Much effort has been devoted to studying (2). Applying the undetermined coefficients method to (2), Zhang et al. [8] presented some explicit kink profile solitary wave solutions. $\mathrm{Li}$ et al. [10] gave some kink profile solitary wave solutions by means of a new auto-Bäclund transformation. Subsequently, Li et al. [11] improved the method presented by Yan and Zhang [12] with a proper transformation. Utilizing the improved method, they obtained some explicit exact solutions. Feng and Knobel [13] made qualitative analysis to (2) and gave the parametric conditions under which there does not exist any bell profile solitary wave solution or periodic traveling wave solution. Furthermore, they used the first integral method to obtain a new kink profile solitary wave solution. By finding a parabola solution connecting two singular points of a planar dynamical system, Li et al. [14] gave the existence conditions of kink profile solitary wave solutions and some exact explicit parametric representations of kink profile solitary wave solutions of (2).

Although a considerable amount of research works has been devoted to (2), there are still some problems which need to be studied further, for example, in addition to kink profile solitary wave solutions, whether (2) has other kinds of bounded traveling wave solutions? As the dissipation effect is varying, how does the shape of bounded traveling wave solutions evolve? In this paper, we will find that, besides kink profile solitary wave solutions, (2) also has damped oscillatory solutions. In addition, we will prove that a bounded traveling wave appears as a kink profile solitary wave if dissipation effect is large, and it appears as a damped oscillatory wave if dissipation effect is small. More importantly, we will discuss how to obtain the approximate damped oscillatory solutions and their error estimates.

The remainder of this paper is organized as follows. In Section 2, the theory and method of planar dynamical systems are applied to study the existence and number of bounded traveling wave solutions of (2). In Section 3, the influence of dissipation on the behavior of bounded traveling wave solutions is studied. It is concluded that the behavior of bounded traveling wave solutions is related to four critical values $r_{1}=-\sqrt{4 b p \beta \phi_{1}\left(\phi_{1}-\phi_{2}\right) /(2 p+1)}, r_{2}=$ $-\sqrt{4 b p \beta \phi_{2}\left(\phi_{2}-\phi_{1}\right) /(2 p+1)}, r_{3}=-\sqrt{-4 \beta c}$, and $r_{4}=$ $-\sqrt{4 p \beta c}$. In Section 4 , according to the evolution relations of orbits in the global phase portraits, the structure of approximate damped oscillatory solutions traveling to the left is designed. And then, by the undetermined coefficients method, we obtain these approximate solutions. To verify the rationality of the approximate damped oscillatory solutions obtained in Section 4, error estimates are studied in Section 5. The results reveal that the errors between exact solutions and approximate solutions are infinitesimal decreasing in the exponential form. In Section 6, a brief conclusion is given.

\section{Existence and Number of Bounded Traveling Wave Solutions}

Assume that (2) has traveling wave solutions of the form $u(x, t)=U(\xi)=U(x-c t)$, where $c$ is the wave speed; then (2) is transformed into the following nonlinear ordinary differential equation:

$$
\begin{aligned}
& -c U^{\prime}(\xi)+a U^{p}(\xi) U^{\prime}(\xi) \\
& \quad+b U^{2 p}(\xi) U^{\prime}(\xi)+r U^{\prime \prime}(\xi)+\beta U^{\prime \prime \prime}(\xi)=0 .
\end{aligned}
$$

Integrating the above equation once with respect to $\xi$ yields

$$
\begin{aligned}
& \beta U^{\prime \prime}(\xi)+r U^{\prime}(\xi)-c U(\xi) \\
& +\frac{a}{p+1} U^{p+1}(\xi)+\frac{b}{2 p+1} U^{2 p+1}(\xi)=g
\end{aligned}
$$

where $g$ is an integral constant. To find traveling wave solutions satisfying

$$
\begin{array}{r}
C_{ \pm}=\lim _{\xi \rightarrow \pm \infty} U(\xi), \\
U^{\prime}(\xi), U^{\prime \prime}(\xi) \longrightarrow 0, \\
|\xi| \longrightarrow+\infty,
\end{array}
$$

where $C_{ \pm}$are the zero roots of the following algebraic equation,

$$
\frac{b}{2 p+1} x^{2 p+1}+\frac{a}{p+1} x^{p+1}-c x=0,
$$

let $|\xi| \rightarrow+\infty$ on both hand sides of (4); then we have $g=0$. Hence, the problem is converted into solving the following ordinary differential equation:

$$
\begin{aligned}
& \beta U^{\prime \prime}(\xi)+r U^{\prime}(\xi)-c U(\xi)+\frac{a}{p+1} U^{p+1}(\xi) \\
& +\frac{b}{2 p+1} U^{2 p+1}(\xi)=0 .
\end{aligned}
$$

Let $\phi=U(\xi)$ and $y=U^{\prime}(\xi)$; then (7) can be equivalently rewritten as the following planar dynamical system:

$$
\begin{aligned}
\frac{d \phi}{d \xi} & =y \triangleq P(\phi, y) \\
\frac{d y}{d \xi} & =-\frac{r}{\beta} y+\frac{c}{\beta} \phi-\frac{a}{(p+1) \beta} \phi^{p+1}-\frac{b}{(2 p+1) \beta} \phi^{2 p+1} \\
& \triangleq Q(\phi, y) .
\end{aligned}
$$

It is well known that the phase orbits defined by the vector fields of system (8) determine all solutions of (7), thereby determining all bounded traveling wave solutions of (2) satisfying (5). Hence, it is necessary to employ the theory and method of planar dynamical systems $[15,16]$ to analyze 
the dynamical behavior of $(8)$ in $(\phi, y)$ phase plane as the parameters are changed. Denote that

$$
\begin{aligned}
f(\phi) & =\frac{b}{2 p+1} \phi^{2 p+1}+\frac{a}{p+1} \phi^{p+1}-c \phi, \\
\Delta & =a^{2}(2 p+1)+4 b c(p+1)^{2}, \\
\bar{\Delta} & =a^{2} p+b c(2 p+1)(p+1), \\
\Delta_{i} & =r^{2}-4 \beta\left(b \phi_{i}^{2}+a \phi_{i}-c\right) \quad(i=0, \ldots, 4), \\
\phi_{0} & =0, \\
\phi_{1,2} & =\frac{-a(2 p+1) \pm \sqrt{(2 p+1) \Delta}}{2 b(p+1)}, \\
\phi_{3} & =-\frac{a(2 p+1)}{2 b(p+1)}, \\
\phi_{4} & =\frac{c(p+1)}{a}, \\
\phi_{i_{ \pm}} & = \pm \sqrt[p]{\phi_{i}}(i=1, \ldots, 4) .
\end{aligned}
$$

Since the number of real roots of $f(\phi)=0$ determines the number of singular points of (8), and at least two singular points determine a bounded orbit, it is easily seen that (8) does not have any bounded orbits under one of the following conditions: (I) $\Delta<0$, (II) $p$ is an even number, $a b>0$ and $b c<0$, and (III) $p$ is an even number, $b=0$ and $a c<0$. Hence, the global phase portraits under the above conditions are neglected in this section. For clarity and nonrepetitiveness, we only present the global phase portraits in the case $a<0$.

(i) $p$ is an even number (see Figures 1-4).

(ii) $p$ is an odd number (see Figures 5-9).

Remark 1. (i) $P_{0}, P_{i_{+}}(i=1,2,3,4), A_{i}(i=1,2)$ in Figures $1-9$ represent the singular point $(0,0)$, singular points $\left(\phi_{i_{+}}, 0\right)(i=1, \ldots, 4)$, and singular points at infinity on $y$-axis, respectively. (ii) When $b>0$, the regions around $A_{i}(i=1,2)$ are hyperbolic type. When $b=0, p$ is an even number, the regions around $A_{i}(i=1,2)$ are elliptic type. When $b=0, p$ is an odd number, the regions around $A_{i}(i=1,2)$ are parabolic type. (iii) When $p$ is an even number, $\beta>0, a>0, b \geq 0$, and $c>0$, the bounded orbits are similar to those shown in Figure 1. When $p$ is an odd number, $a>0$, the bounded orbits are similar to those shown in Figures 5-9. (iv) When $p$ is an even number, $\beta>0, a<0, b>0, c<0$, and $\Delta>0$, there exist four possible global phase portraits described by Figures 3(a), 3(b), 3(c), and 3(d). If $\bar{\Delta}>0$, then only Figures 3(a), $3(\mathrm{~b})$, and $3(\mathrm{~d})$ describe the orbit distribution. If $\bar{\Delta}=0$, then only Figures 3(a) and 3(d) describe the orbit distribution. If $\bar{\Delta}<0$, then only Figures 3(a), 3(c), and 3(d) describe the orbit distribution. Figures 6(a), 6(b), 6(c), and 6(d) can be explained similarly.

In addition to Figures 1-9 shown above, we have the following theorem.

Theorem 2. (i) When $\beta>0, r<0, p$ is an even number, and $a, b, c$, and $p$ satisfy none of the following conditions: (I) $\Delta=a^{2}(2 p+1)+4 b c(p+1)^{2}<0$, (II) $a b>0$ and $b c<$ 0 , and (III) $b=0$ and $a c<0$, (2) has either two bounded traveling wave solutions or four bounded traveling wave solutions.

(ii) When $\beta>0, r<0, p$ is an odd number, and $a, b, c$, and $p$ do not satisfy $\Delta=a^{2}(2 p+1)+4 b c(p+1)^{2}<0$, (2) has either one bounded traveling wave solution or two bounded traveling wave solutions.

\section{Behavior of Bounded Traveling Wave Solutions}

To study dissipation effect on behavior of bounded traveling wave solutions, we denote that

$$
\begin{aligned}
& r_{1}=-\sqrt{\frac{4 b p \beta \phi_{1}\left(\phi_{1}-\phi_{2}\right)}{2 p+1},} \\
& r_{2}=-\sqrt{\frac{4 b p \beta \phi_{2}\left(\phi_{2}-\phi_{1}\right)}{2 p+1}}, \\
& r_{3}=-\sqrt{-4 \beta c} \\
& r_{4}=-\sqrt{4 p \beta c}
\end{aligned}
$$

and quote the following lemma [17-19].

Lemma 3. Assume that $f \in C^{1}[0,1], f(0)=f(1)=0$, $f^{\prime}(0)>0, f^{\prime}(1)<0$, and for all $u \in(0,1), f(u)>0$ holds. Then, there exists $r^{*}$ satisfying

$$
-2 \sqrt{\sup \frac{f(u)}{u}} \leq r^{*} \leq-2 \sqrt{f^{\prime}(0)},
$$

such that the necessary and sufficient condition under which problem

$$
\begin{array}{r}
u^{\prime \prime}+r u^{\prime}+f(u)=0, \\
u(-\infty)=0, \\
u(+\infty)=1
\end{array}
$$

has a monotone solution is $r \leq r^{*}$.

By the above lemma, we can prove the following theorems. 


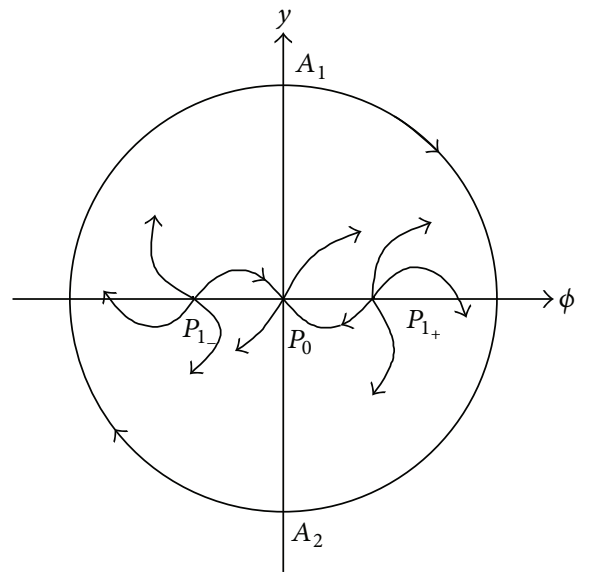

(a) $\left(\Delta_{1}>0\right)$

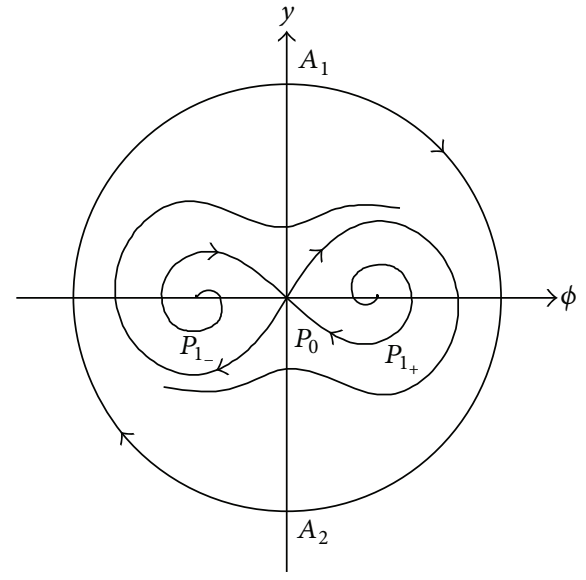

(b) $\left(\Delta_{1}<0\right)$

Figure 1: $(\beta>0, a<0, b>0, c>0)$.

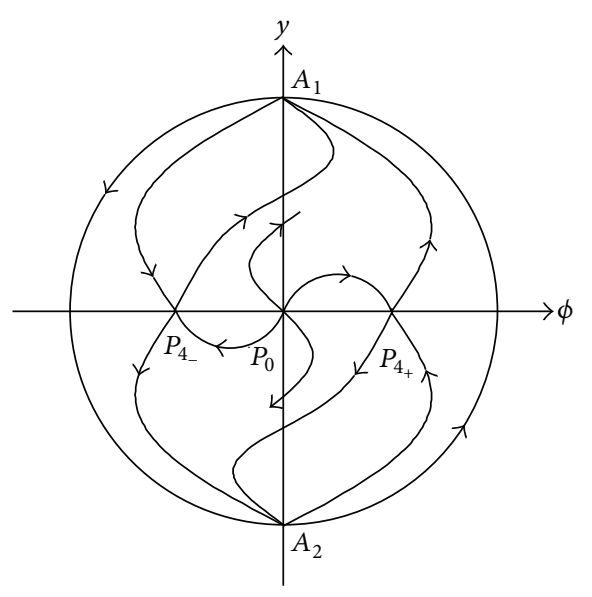

(a) $\left(\Delta_{0}>0\right)$

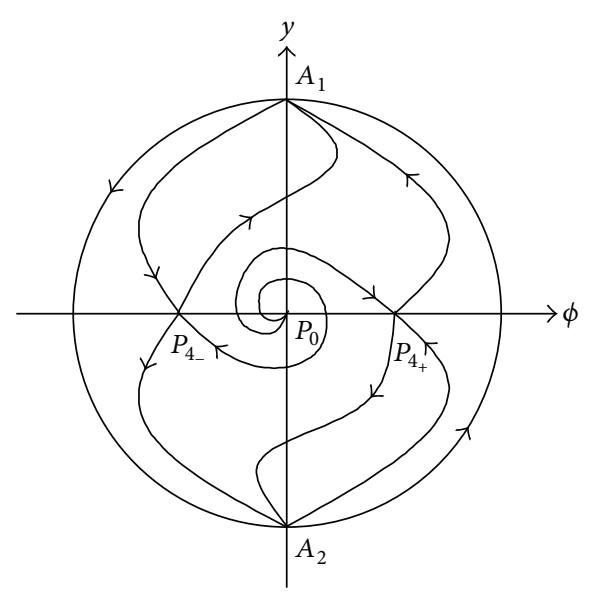

(b) $\left(\Delta_{0}<0\right)$

Figure 2: $(\beta>0, a<0, b=0, c<0)$.

Theorem 4. Suppose that $\beta>0, p$ is an even number, $a<0$, $b>0$, and $c>0$.

(i) When $r<r_{1}$, (2) has a monotone decreasing kink profile solitary wave solution $U(\xi)$ satisfying $U(-\infty)=$ $\sqrt[p]{\phi_{1}}, U(+\infty)=0$, and a monotone increasing kink profile solitary wave solution $U(\xi)$ satisfying $U(-\infty)=$ $-\sqrt[p]{\phi_{1}}, U(+\infty)=0$. These solutions correspond to the orbits $L\left(P_{1_{+}}, P_{0}\right)$ in Figure $1(a)$, respectively.

(ii) When $r_{1}<r<0$, (2) has two oscillatory traveling wave solutions $U(\xi)$. One satisfies $U(-\infty)=\sqrt[p]{\phi_{1}}, U(+\infty)=$ 0 , and the other satisfies $U(-\infty)=-\sqrt[p]{\phi_{1}}, U(+\infty)=$ 0 . These solutions correspond to the orbits $L\left(P_{1_{ \pm}}, P_{0}\right)$ in Figure 1(b), respectively.

Proof. (i) Substituting the transformation

$$
V(\xi)=\frac{U(\xi)+\sqrt[p]{\phi_{1}}}{2 \sqrt[p]{\phi_{1}}}
$$

into (7), it is obtained that

$$
\begin{gathered}
\beta V^{\prime \prime}(\xi)+r V^{\prime}(\xi)+\frac{2^{p} b \phi_{1}}{2 p+1}\left(V(\xi)-\frac{1}{2}\right) \\
\cdot\left(\left(V(\xi)-\frac{1}{2}\right)^{p}-\left(\frac{1}{2}\right)^{p}\right) \\
\cdot\left(2^{p} \phi_{1}\left(V(\xi)-\frac{1}{2}\right)^{p}-\phi_{2}\right)=0 .
\end{gathered}
$$

Evidently, $(0,0),(1 / 2,0)$, and $(1,0)$ corresponding to $P_{1_{-}}\left(-\sqrt[p]{\phi_{1}}, 0\right), P_{0}(0,0)$, and $P_{1_{+}}\left(\sqrt[p]{\phi_{1}}, 0\right)$ are the singular points of the planar dynamical system associated with (14). It can be proved that the properties of $(0,0),(1 / 2,0)$, and $(1,0)$ are the same as those of $P_{1_{-}}, P_{0}$, and $P_{1_{+}}$, and the results obtained in Section 2 also hold for (14). Since when $p$ is an 


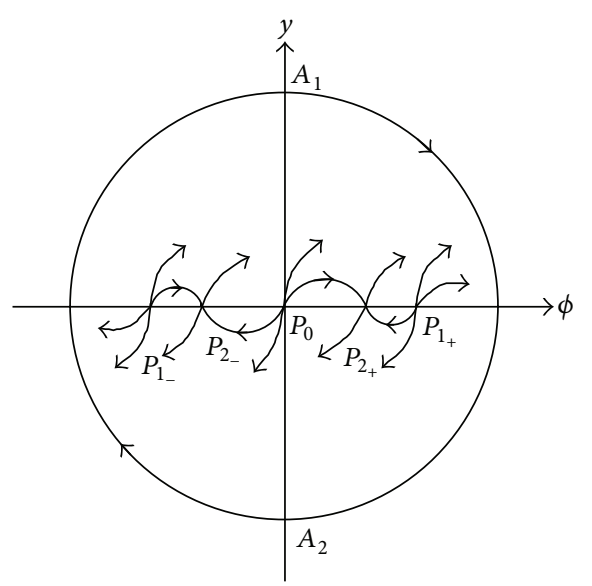

(a) $\left(\Delta_{0}>0, \Delta_{1}>0\right)$

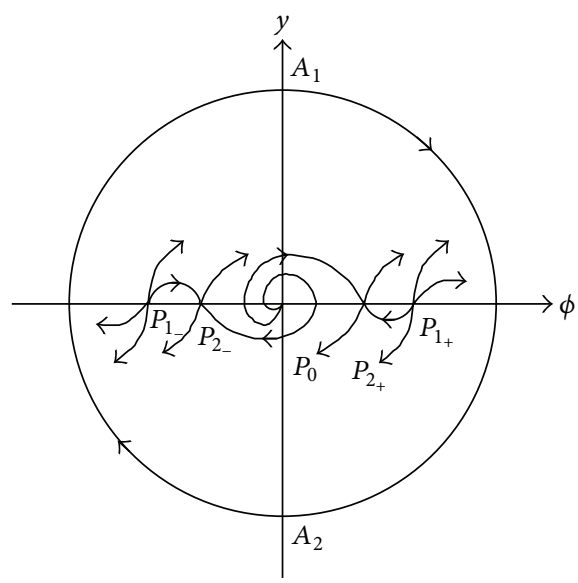

(c) $\left(\Delta_{0}<0, \Delta_{1}>0\right)$

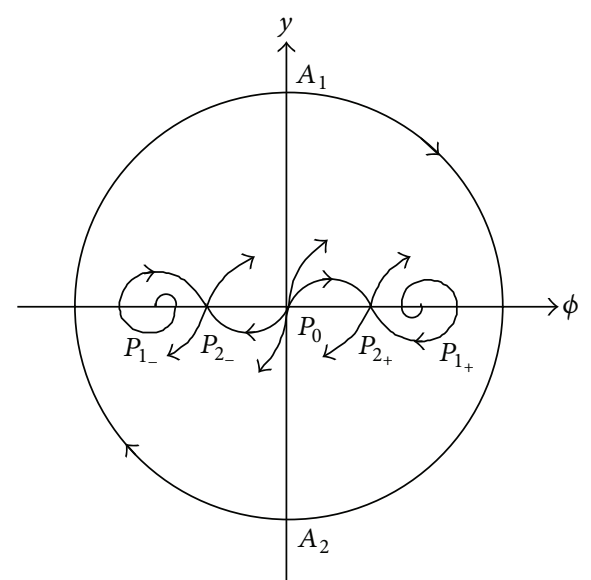

(b) $\left(\Delta_{0}>0, \Delta_{1}<0\right)$

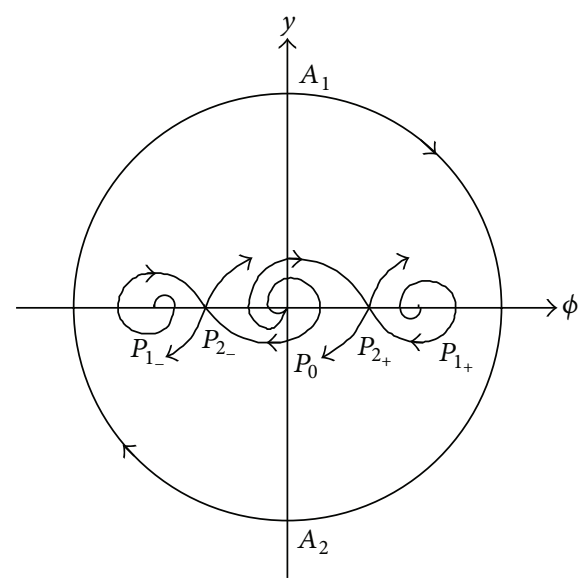

(d) $\left(\Delta_{0}<0, \Delta_{1}<0\right)$

Figure 3: $(\beta>0, a<0, b>0, c<0, \Delta>0)$.

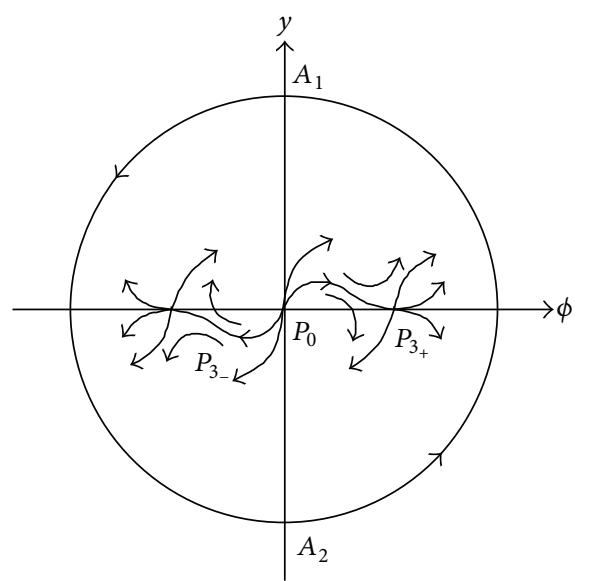

(a) $\left(\Delta_{0}>0\right)$

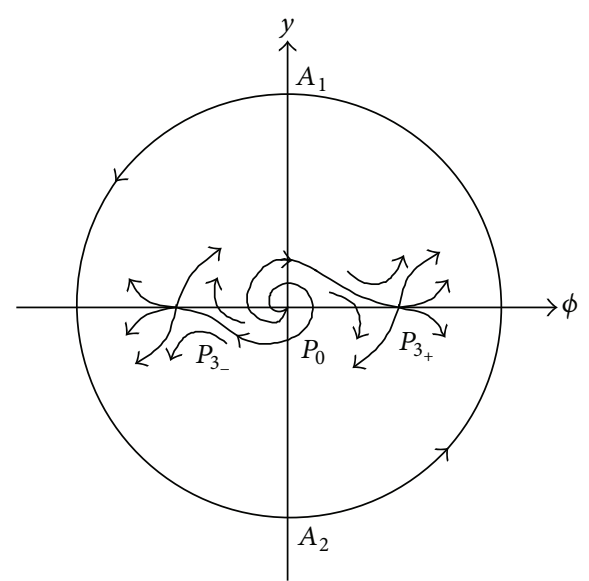

(b) $\left(\Delta_{0}<0\right)$

Figure 4: $(\beta>0, a<0, b>0, c<0, \Delta=0)$. 


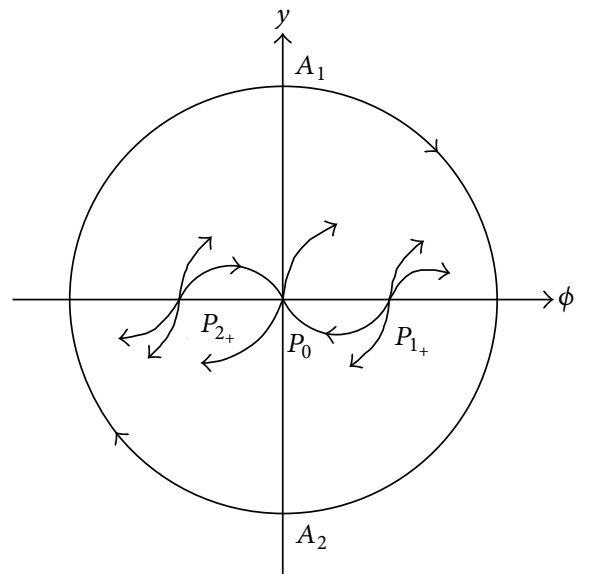

(a) $\left(\Delta_{2}>\Delta_{1}>0\right)$

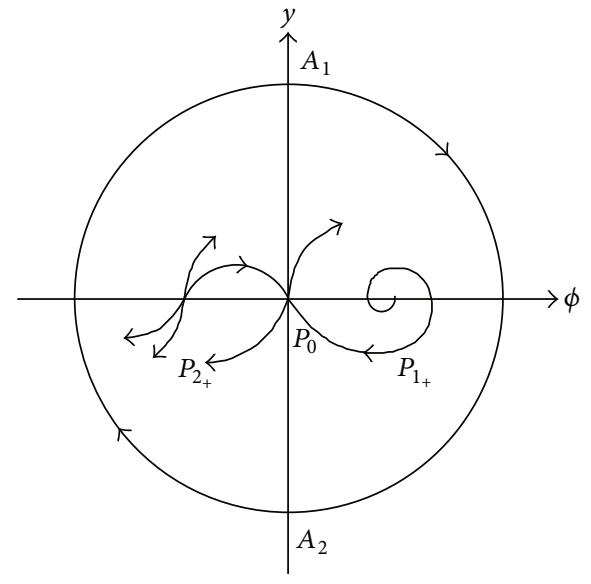

(b) $\left(\Delta_{2}>0>\Delta_{1}\right)$

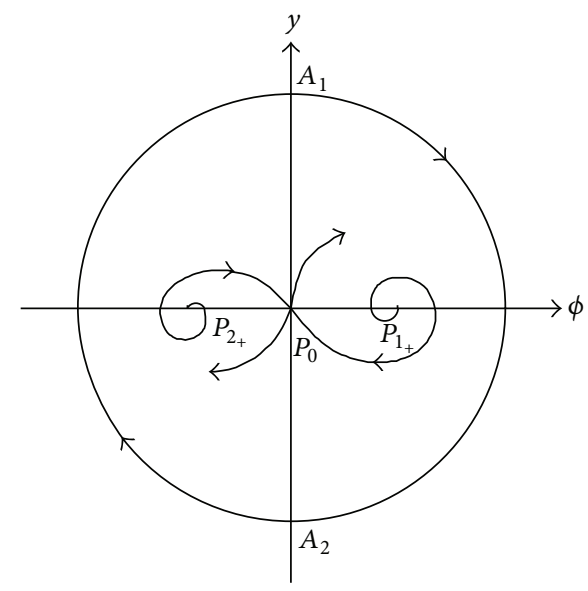

(c) $\left(\Delta_{1}<\Delta_{2}<0\right)$

Figure 5: $(\beta>0, a<0, b>0, c>0)$.

even number, $a<0, b>0, c>0$, and $r<r_{1}$, (7) only has two bounded solutions satisfying
(A) $U(-\infty)=\sqrt[p]{\phi_{1}}, U(+\infty)=0$,
(B) $U(-\infty)=-\sqrt[p]{\phi_{1}}, U(+\infty)=0$,

respectively, (14) must only have two bounded solutions satisfying

$$
\begin{aligned}
& \left(\mathrm{A}^{\prime}\right) V(-\infty)=1, V(+\infty)=1 / 2, \\
& \left(\mathrm{~B}^{\prime}\right) V(-\infty)=0, V(+\infty)=1 / 2,
\end{aligned}
$$

respectively.

Using the transformation $W(\xi)=2(1-V(\xi))$, (14) becomes

$$
\begin{gathered}
W^{\prime \prime}(\xi)+\frac{r}{\beta} W^{\prime}(\xi)+\frac{b \phi_{1}}{\beta(2 p+1)} \\
\cdot(W(\xi)-1)\left((W(\xi)-1)^{p}-1\right) \\
\cdot\left(\phi_{1}(W(\xi)-1)^{p}-\phi_{2}\right)=0 .
\end{gathered}
$$

Therefore, the solution of (14) satisfying $\left(\mathrm{A}^{\prime}\right)$ corresponds to the solution of (15) satisfying

$$
\begin{aligned}
& W(-\infty)=0, \\
& W(+\infty)=1 .
\end{aligned}
$$

Let $F(W)=\left(b \phi_{1} / \beta(2 p+1)\right)(W-1)\left((1-W)^{p}-1\right)\left(\phi_{1}(1-W)^{p}-\right.$ $\left.\phi_{2}\right)$ for all $W \in(0,1)$; then we have $F^{\prime}(W)=\left(b \phi_{1} / \beta(2 p+\right.$ $1))\left(\left((1+p)(1-W)^{p}-1\right)\left(\phi_{1}(1-W)^{p}-\phi_{2}\right)+\phi_{1} p(1-W)^{p}((1-\right.$ $\left.\left.W)^{p}-1\right)\right)$. Since $F(0)=F(1)=0, F^{\prime}(0)=\left(b p \phi_{1} / \beta(2 p+\right.$ 1)) $\left(\phi_{1}-\phi_{2}\right)>0, F^{\prime}(1)=b \phi_{1} \phi_{2} / \beta(2 p+1)<0$, and $F(W)>0$ for all $W \in(0,1)$, from Lemma 3 , there exists $r^{*}$ satisfying

$$
-2 \sqrt{\sup \frac{F(W)}{W}} \leq r^{*} \leq-2 \sqrt{F^{\prime}(0)}
$$

such that, when $r \leq \beta r^{*}$, (15) has a monotone solution satisfying (16). 


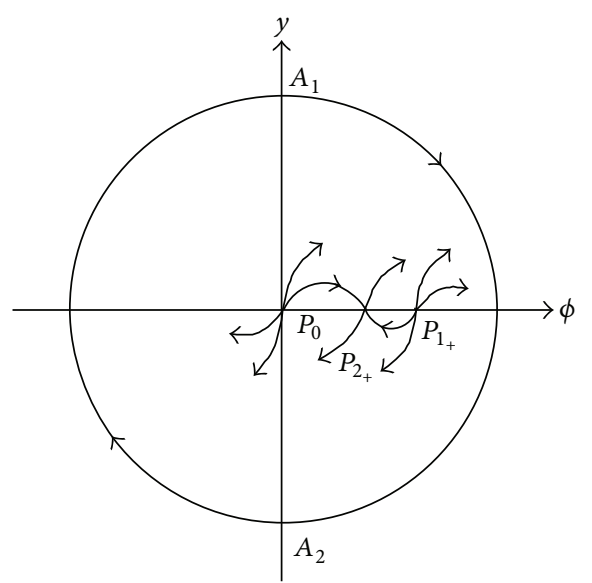

(a) $\left(\Delta_{0}>0, \Delta_{1}>0\right)$

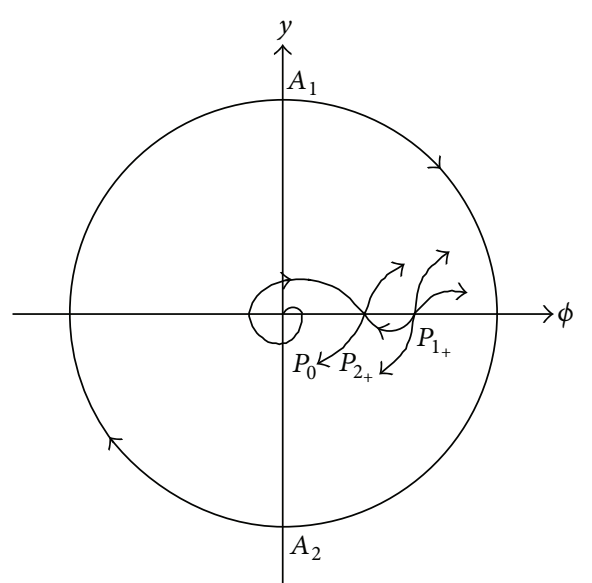

(c) $\left(\Delta_{0}<0, \Delta_{1}>0\right)$

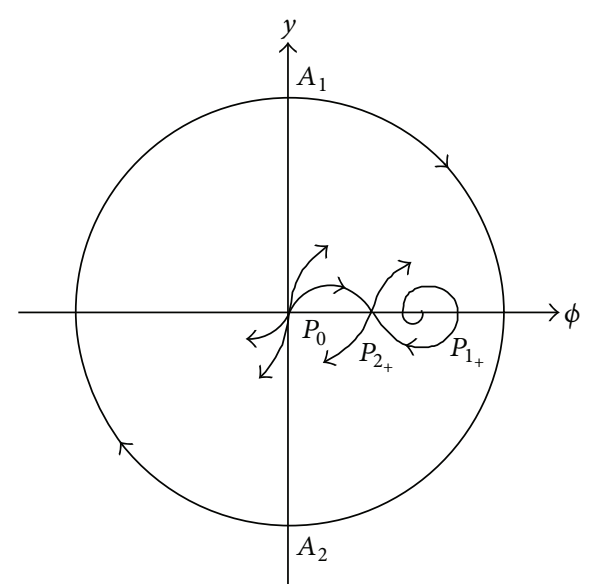

(b) $\left(\Delta_{0}>0, \Delta_{1}<0\right)$

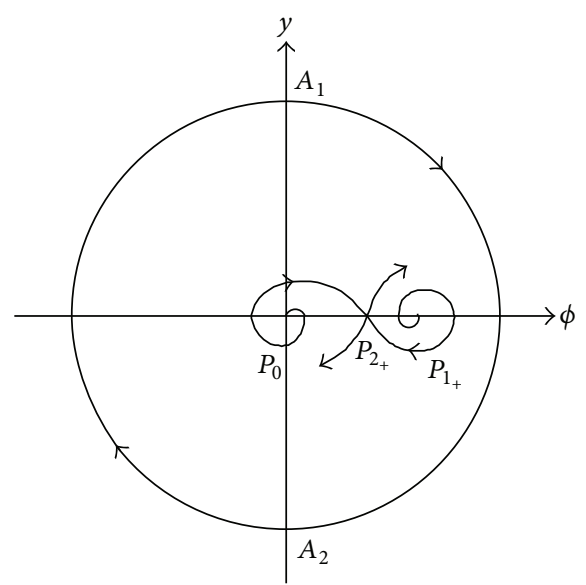

(d) $\left(\Delta_{0}<0, \Delta_{1}<0\right)$

Figure 6: $(\beta>0, a<0, b>0, c<0, \Delta>0)$.

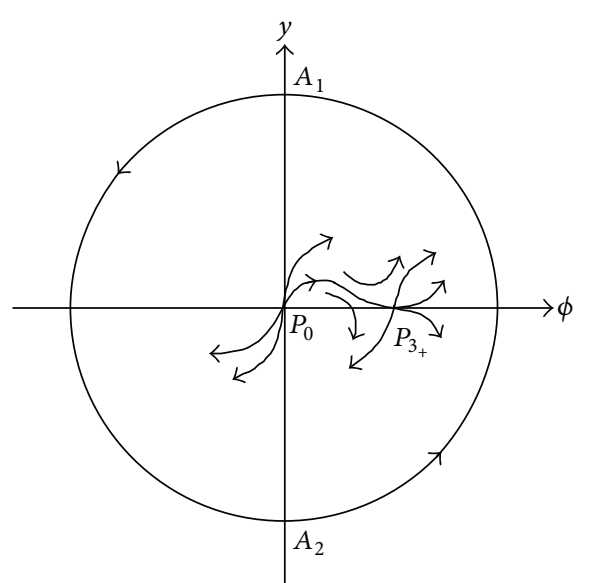

(a) $\left(\Delta_{0}>0\right)$

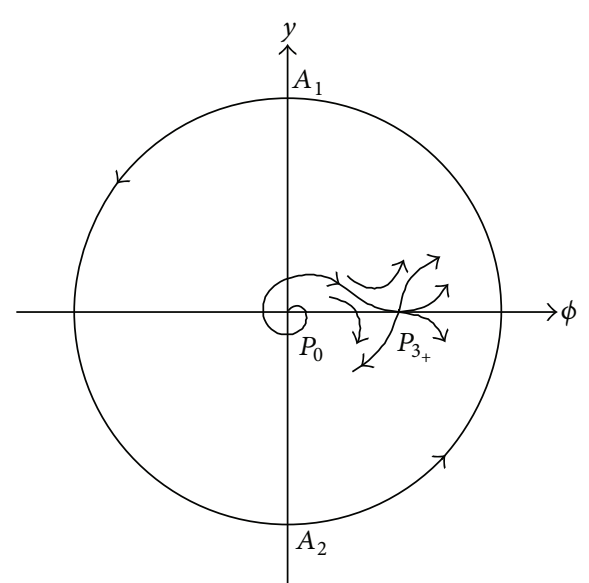

(b) $\left(\Delta_{0}<0\right)$

Figure 7: $(\beta>0, a<0, b>0, c<0, \Delta=0)$. 


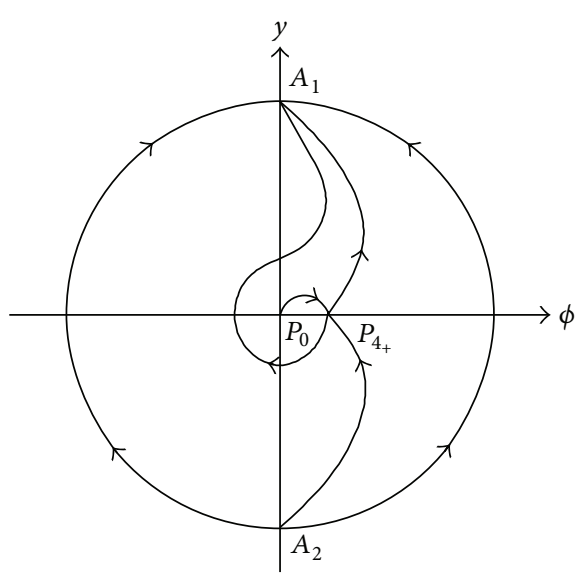

(a) $\left(\Delta_{0}>0\right)$

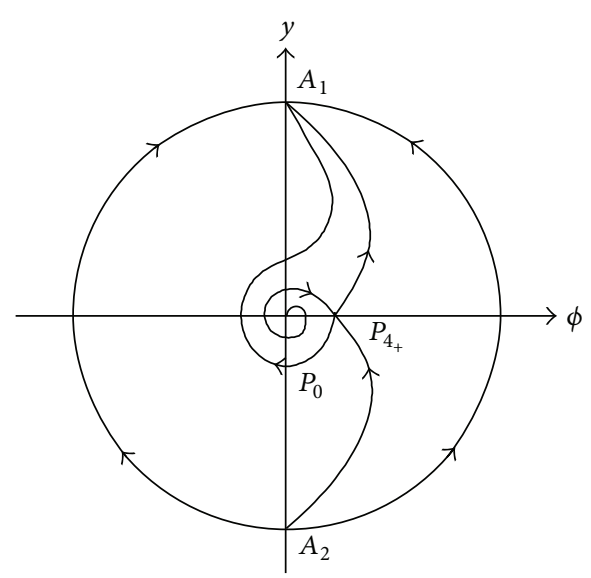

(b) $\left(\Delta_{0}<0\right)$

Figure 8: $(\beta>0, a<0, b=0, c<0)$.

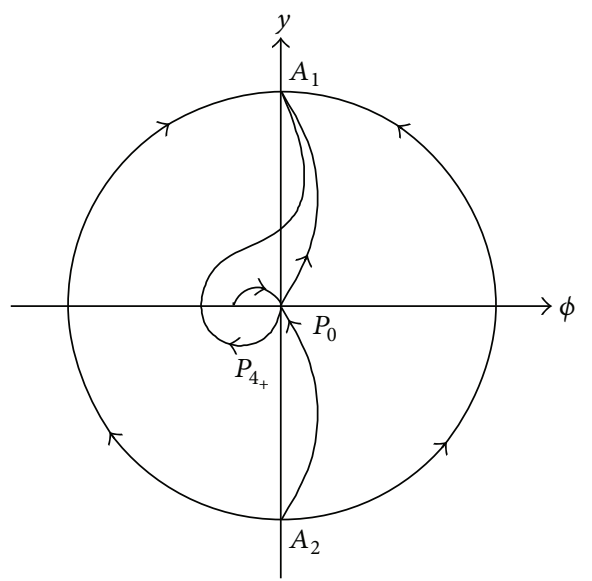

(a) $\left(\Delta_{4}>0\right)$

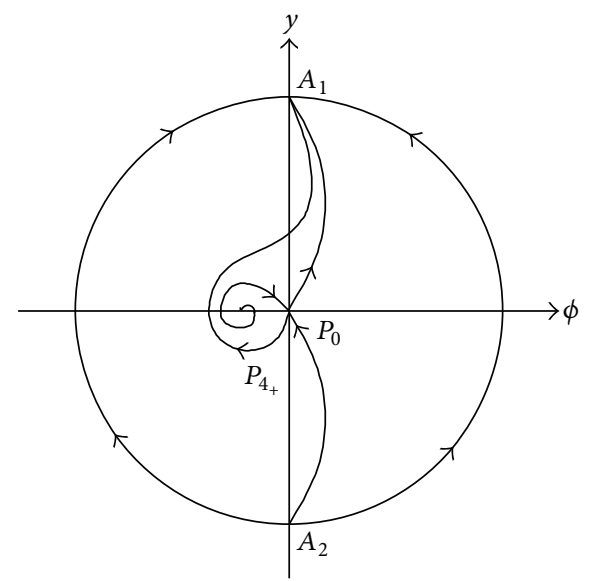

(b) $\left(\Delta_{4}<0\right)$

Figure 9: $(\beta>0, a<0, b=0, c>0)$.

$\forall W \in(0,1)$, we have

$$
\begin{aligned}
\left(\frac{F(W)}{W}\right)^{\prime} & \\
= & \frac{b \phi_{1}}{\beta(2 p+1)} \\
& \cdot\left(\frac{g(W)}{W^{2}}\left(\phi_{1}(1-W)^{p}-\phi_{2}\right)\right. \\
& \left.+\frac{p \phi_{1}(1-W)^{p}\left((1-W)^{p}-1\right)}{W}\right),
\end{aligned}
$$

where $g(W)=(1-W)^{p}(p W+1)-1$. Since $g^{\prime}(W)=-p(p+$ $1) W(1-W)^{p-1}<0$ holds for all $W \in(0,1)$ and $g(0)=0$, we have $g(W)<0$ for all $W \in(0,1)$. Consequently, we have $(F(W) / W)^{\prime}<0$ for all $W \in(0,1)$, which indicates that
$F(W) / W$ monotonically decreases in $(0,1)$. Furthermore, we have

$$
\begin{aligned}
\operatorname{up} \frac{F(W)}{W} & =\lim _{W \rightarrow 0} \frac{F(W)}{W}=\lim _{W \rightarrow 0} F^{\prime}(W) \\
& =\frac{b p \phi_{1}}{\beta(2 p+1)}\left(\phi_{1}-\phi_{2}\right) .
\end{aligned}
$$

Combining (19) with (17), we obtain $r^{*}=$ $-\sqrt{\left(4 b p \phi_{1} / \beta(2 p+1)\right)\left(\phi_{1}-\phi_{2}\right)}$. Therefore, from Lemma 3, it is concluded that when $r \leq \beta r^{*}=r_{1}$, (15) has a monotone increasing solution. According to the relation between $W(\xi)$ and $U(\xi)$, it is easily seen that when $r \leq r_{1}$, (2) has a monotone decreasing kink profile solitary wave solution satisfying (A).

Now, let us consider the solution of (14) satisfying $\left(\mathrm{B}^{\prime}\right)$. Substituting the transformation $W(\xi)=2 V(\xi)$ into (14), we obtain (15) as well. Therefore, the solution of (14) satisfying $\left(\mathrm{B}^{\prime}\right)$ corresponds to the solution of (15) satisfying (16). 
Similarly, we can prove that when $r \leq r_{1}$, (15) has a monotone increasing solution. According to the relation between $W(\xi)$ and $U(\xi)$, it is easily seen that (2) has a monotone increasing kink profile solitary wave solution satisfying (B).

(ii) By the theory and method of planar dynamical systems, it is easily obtained that when $r_{1}<r<0$, there exist an orbit $L\left(P_{1_{+}}, P_{0}\right)$ connecting the unstable focus $P_{1_{+}}$and the saddle point $P_{0}$ in $\{(\phi, y) \mid \phi>0,-\infty<y<+\infty\}$ and an orbit $L\left(P_{1_{-}}, P_{0}\right)$ connecting the unstable focus $P_{1_{-}}$and the saddle point $P_{0}$ in $\{(\phi, y) \mid \phi<0,-\infty<y<+\infty\}$. Owing to the fact that the orbits $L\left(P_{1_{+}}, 0\right)$ tend to $P_{1_{+}}$spirally as $\xi \rightarrow$ $-\infty$, the corresponding bounded traveling wave solutions $U(\xi)$ are oscillatory. One satisfies $U(-\infty)=\sqrt[p]{\phi_{1}}, U(+\infty)=0$, and the other satisfies $U(-\infty)=-\sqrt[p]{\phi_{1}}, U(+\infty)=0$.

Remark 5. $L(P, Q)$ denotes an orbit whose $\alpha$ limit set is $P$, and $\omega$ limit set is $Q$.

Similar to Theorem 4, the following theorems can be proved.

Theorem 6. Suppose that $\beta>0, p$ is an even number, $a<0$, $b>0,-a^{2}(2 p+1) / 4 b(p+1)^{2}<c<0$.

(i) When $r<r_{1}$, (2) has a monotone decreasing kink profile solitary wave solution $U(\xi)$ satisfying $U(-\infty)=$ $\sqrt[p]{\phi_{1}}, U(+\infty)=\sqrt[p]{\phi_{2}}$, and a monotone increasing kink profile solitary wave solution $U(\xi)$ satisfying $U(-\infty)=$ $-\sqrt[p]{\phi_{1}}, U(+\infty)=-\sqrt[p]{\phi_{2}}$. These solutions correspond to the orbits $L\left(P_{1_{ \pm}}, P_{2_{ \pm}}\right)$in Figures $3(a)$ and $3(c)$, respectively.

(ii) When $r_{1}<r<0$, (2) has two oscillatory traveling wave solutions $U(\xi)$. One satisfies $U(-\infty)=\sqrt[p]{\phi_{1}}$, $U(+\infty)=\sqrt[p]{\phi_{2}}$, and the other satisfies $U(-\infty)=$ $-\sqrt[p]{\phi_{1}}, U(+\infty)=-\sqrt[p]{\phi_{2}}$. These solutions correspond to the orbits $L\left(P_{1_{ \pm}}, P_{2_{ \pm}}\right)$in Figures $3(b)$ and $3(d)$, respectively.

(iii) When $r<r_{3}$, (2) has a monotone increasing kink profile solitary wave solution $U(\xi)$ satisfying $U(-\infty)=$ $0, U(+\infty)=\sqrt[p]{\phi_{2}}$, and a monotone decreasing kink profile solitary wave solution $U(\xi)$ satisfying $U(-\infty)=$ $0, U(+\infty)=-\sqrt[p]{\phi_{2}}$. These solutions correspond to the orbits $L\left(P_{0}, P_{2_{ \pm}}\right)$in Figures $3(a)$ and $3(b)$, respectively.

(iv) When $r_{3}<r<0$, (2) has two oscillatory traveling wave solutions $U(\xi)$. One satisfies $U(-\infty)=0$, $U(+\infty)=\sqrt[p]{\phi_{2}}$, and the other satisfies $U(-\infty)=0$, $U(+\infty)=-\sqrt[p]{\phi_{2}}$. These solutions correspond to the orbits $L\left(P_{0}, P_{2_{ \pm}}\right)$in Figures $3(c)$ and $3(d)$, respectively.

Theorem 7. Suppose that $\beta>0, p$ is an even number, $a<0$, $b>0, c=-a^{2}(2 p+1) / 4 b(p+1)^{2}$.

(i) When $r<r_{3}$, (2) has a monotone increasing kink profile solitary wave solution $U(\xi)$ satisfying $U(-\infty)=$ $0, U(+\infty)=\sqrt[p]{\phi_{3}}$, and a monotone decreasing kink profile solitary wave solution $U(\xi)$ satisfying $U(-\infty)=$ $0, U(+\infty)=-\sqrt[p]{\phi_{3}}$. These solutions correspond to the orbits $L\left(P_{0}, P_{3_{+}}\right)$in Figure 4(a), respectively. (ii) When $r_{3}<r<0$, (2) has two oscillatory traveling wave solutions $U(\xi)$. One satisfies $U(-\infty)=0$, $U(+\infty)=\sqrt[p]{\phi_{3}}$, and the other satisfies $U(-\infty)=0$, $U(+\infty)=-\sqrt[p]{\phi_{3}}$. These solutions correspond to the orbits $L\left(P_{0}, P_{3_{ \pm}}\right)$in Figure $4(b)$, respectively.

Theorem 8. Suppose that $\beta>0, p$ is an even number, $a>0$, $b=0$, and $c>0$.

(i) When $r<r_{4}$, (2) has a monotone decreasing kink profile solitary wave solution $U(\xi)$ satisfying $U(-\infty)=$ $\sqrt[p]{\phi_{4}}, U(+\infty)=0$, and a monotone increasing kink profile solitary wave solution $U(\xi)$ satisfying $U(-\infty)=$ $-\sqrt[p]{\phi_{4}}, U(+\infty)=0$

(ii) When $r_{4}<r<0$, (2) has two oscillatory traveling wave solutions $U(\xi)$. One satisfies $U(-\infty)=\sqrt[p]{\phi_{4}}, U(+\infty)=$ 0 , and the other satisfies $U(-\infty)=-\sqrt[p]{\phi_{4}}, U(+\infty)=0$.

Theorem 9. Suppose that $\beta>0, p$ is an even number, $a<0$, $b=0$, and $c<0$.

(i) When $r<r_{3}$, (2) has a monotone increasing kink profile solitary wave solution $U(\xi)$ satisfying $U(-\infty)=$ $0, U(+\infty)=\sqrt[p]{\phi_{4}}$, and a monotone decreasing kink profile solitary wave solution $U(\xi)$ satisfying $U(-\infty)=$ $0, U(+\infty)=-\sqrt[p]{\phi_{4}}$. These solutions correspond to the orbits $L\left(P_{0}, P_{4_{+}}\right)$in Figure 2(a), respectively.

(ii) When $r_{3}<r<0$, (2) has two oscillatory traveling wave solutions $U(\xi)$. One satisfies $U(-\infty)=0$, $U(+\infty)=\sqrt[p]{\phi_{4}}$, and the other satisfies $U(-\infty)=0$, $U(+\infty)=-\sqrt[p]{\phi_{4}}$. These solutions correspond to the orbits $L\left(P_{0}, P_{4_{+}}\right)$in Figure 2(b), respectively.

Theorem 10. Suppose that $\beta>0, p$ is an odd number, $a<0$, $b>0$, and $c>0$.

(i) When $r<r_{1}$, (2) has a monotone decreasing kink profile solitary wave solution $U(\xi)$ satisfying $U(-\infty)=$ $\sqrt[p]{\phi_{1}}, U(+\infty)=0$, which corresponds to the orbit $L\left(P_{1_{+}}\right.$, $\left.P_{0}\right)$ in Figure 5(a)

(ii) When $r_{1}<r<0$, (2) has an oscillatory traveling wave solution $U(\xi)$ satisfying $U(-\infty)=\sqrt[p]{\phi_{1}}, U(+\infty)=0$, which corresponds to the orbit $L\left(P_{1_{+}}, P_{0}\right)$ in Figures 5(b) and 5(c).

(iii) When $r<r_{2}$, (2) has a monotone increasing kink profile solitary wave solution $U(\xi)$ satisfying $U(-\infty)=$ $\sqrt[p]{\phi_{2}}, U(+\infty)=0$, which corresponds to the orbit $L\left(P_{2_{+}}\right.$, $\left.P_{0}\right)$ in Figures 5(a) and 5(b).

(iv) When $r_{2}<r<0$, (2) has an oscillatory traveling wave solution $U(\xi)$ satisfying $U(-\infty)=\sqrt[p]{\phi_{2}}, U(+\infty)=0$, which corresponds to the orbit $L\left(P_{2_{+}}, P_{0}\right)$ in Figure 5(c).

Theorem 11. Suppose that $\beta>0, p$ is an odd number, $a>0$, $b>0,-a^{2}(2 p+1) / 4 b(p+1)^{2}<c<0$.

(i) When $r<r_{2}$, (2) has a monotone increasing kink profile solitary wave solution $U(\xi)$ satisfying $U(-\infty)=$ $\sqrt[p]{\phi_{2}}, U(+\infty)=\sqrt[p]{\phi_{1}}$. 
(ii) When $r_{2}<r<0$, (2) has an oscillatory traveling wave solution $U(\xi)$ satisfying $U(-\infty)=\sqrt[p]{\phi_{2}}, U(+\infty)=$ $\sqrt[p]{\phi_{1}}$.

(iii) When $r<r_{3}$, (2) has a monotone decreasing kink profile solitary wave solution $U(\xi)$ satisfying $U(-\infty)=$ $0, U(+\infty)=\sqrt[p]{\phi_{1}}$.

(iv) When $r_{3}<r<0$, (2) has an oscillatory traveling wave solution $U(\xi)$ satisfying $U(-\infty)=0, U(+\infty)=\sqrt[p]{\phi_{1}}$.

Theorem 12. Suppose that $\beta>0, p$ is an odd number, $a>0$, $b>0$, and $c=-a^{2}(2 p+1) / 4 b(p+1)^{2}$.

(i) When $r<r_{3}$, (2) has a monotone decreasing kink profile solitary wave solution $U(\xi)$ satisfying $U(-\infty)=$ $0, U(+\infty)=\sqrt[p]{\phi_{3}}$.

(ii) When $r_{3}<r<0$, (2) has an oscillatory traveling wave solution $U(\xi)$ satisfying $U(-\infty)=0, U(+\infty)=\sqrt[p]{\phi_{3}}$.

Theorem 13. Suppose that $\beta>0, p$ is an odd number, $a<0$, $b=0$, and $c>0$.

(i) When $r<r_{4}$, (2) has a monotone increasing kink profile solitary wave solution $U(\xi)$ satisfying $U(-\infty)=$ $\sqrt[p]{\phi_{4}}, U(+\infty)=0$, which corresponds to the orbit $L\left(P_{4_{+}}, P_{0}\right)$ in Figure $9(a)$.

(ii) When $r_{4}<r<0$, (2) has an oscillatory traveling wave $U(\xi)$ satisfying $U(-\infty)=\sqrt[p]{\phi_{4}}, U(+\infty)=0$, which corresponds to the orbit $L\left(P_{4_{+}}, P_{0}\right)$ in Figure $9(b)$.

Theorem 14. Suppose that $\beta>0, p$ is an odd number, $a>0$, $b=0$, and $c<0$.

(i) When $r<r_{3}$, (2) has a monotone decreasing kink profile solitary wave $U(\xi)$ satisfying $U(-\infty)=0$, $U(+\infty)=\sqrt[p]{\phi_{4}}$.

(ii) When $r_{3}<r<0$, (2) has an oscillatory traveling wave $U(\xi)$ satisfying $U(-\infty)=0, U(+\infty)=\sqrt[p]{\phi_{4}}$.

Remark 15. (i) When $a<0$ in Theorems 4 and 10 is changed into $a>0$, the similar conclusions can be established. (ii) It is easily proved that the bounded orbits shown in Figures 6 and 7 are just the right ones shown in Figures 3 and 4 . Therefore, parts of conclusions in Theorems 6 and 7 hold when $p$ is an odd number, $a<0, b>0$, and $-a^{2}(2 p+1) / 4 b(p+1)^{2} \leq$ $c<0$. (iii) When $b=0$ and $a c>0$, the bounded orbits obtained as $p$ is an even number include those obtained as $p$ is an odd number. Hence, similar to Theorems 8 and 9, the relations between the behaviors of the bounded traveling wave solutions and $r$ are obtained.

The above theorems indicate that when $r$ is less than one of the critical values $r_{i}(i=1, \ldots, 4)$, (2) has a bounded traveling wave appearing as a kink profile solitary wave, and when $r$ is more than one of the above critical values, (2) has a bounded traveling wave appearing as an oscillatory traveling wave. In fact, the oscillatory traveling waves also have damped property. To this end, we take those corresponding to the focus-saddle orbits $L\left(P_{1_{ \pm}}, P_{0}\right)$ in Figure $1(\mathrm{~b})$ as examples.
The oscillatory traveling wave solution with damped property is called a damped oscillatory solution in this paper.

Theorem 16. Suppose that $\beta>0, p$ is an even number, $a<0$, $b>0$, and $c>0$. If $r_{1}<r<0$, then (2) has two oscillatory traveling wave solutions $U(\xi)$. (i) The one corresponding to the orbit $L\left(P_{1_{-}}, 0\right)$ in Figure $1(b)$ has minimum at $\check{\xi}_{1}$. Moreover, it has monotonically increasing property at the right hand side of $\check{\xi}_{1}$ and has damped property at the left hand side of $\check{\xi}_{1}$. Namely, there exist numerably infinite maximum points $\widehat{\xi}_{i}(i=$ $1,2, \ldots,+\infty)$ and minimum points $\check{\xi}_{i}(i=1,2, \ldots,+\infty)$ on $\xi$-axis, such that

$$
\begin{aligned}
-\infty<\cdots<\widehat{\xi}_{n}<\check{\xi}_{n}<\cdots<\widehat{\xi}_{1}<\check{\xi}_{1}<+\infty, \\
\lim _{n \rightarrow \infty} \widehat{\xi}_{n}=\lim _{n \rightarrow \infty} \check{\xi}_{n}=-\infty, \\
U\left(\check{\xi}_{1}\right)<\cdots<U\left(\check{\xi}_{n}\right)<\cdots<U(-\infty)<\cdots<U\left(\widehat{\xi}_{n}\right) \\
<\cdots<U\left(\widehat{\xi}_{1}\right)<U(+\infty), \\
\lim _{n \rightarrow \infty} U\left(\widehat{\xi}_{n}\right)=\lim _{n \rightarrow \infty} U\left(\check{\xi}_{n}\right)=U(-\infty), \\
\lim _{n \rightarrow \infty}\left(\widehat{\xi}_{n}-\widehat{\xi}_{n+1}\right)=\lim _{n \rightarrow \infty}\left(\check{\xi}_{n}-\check{\xi}_{n+1}\right) \\
=\frac{4 \pi \beta}{\sqrt{4 \beta\left(b \phi_{1}^{2}+a \phi_{1}-c\right)-r^{2}}} .
\end{aligned}
$$

(ii) The one corresponding to the orbit $L\left(P_{1_{+}}, 0\right)$ in Figure $1(b)$ has maximum at $\widehat{\xi}_{1}$. Moreover, it has monotonically decreasing property at the right hand side of $\widehat{\xi}_{1}$ and has damped property at the left hand side of $\widehat{\xi}_{1}$. Namely, there exist countably infinite maximum points $\widehat{\xi}_{i}(i=1,2, \ldots,+\infty)$ and minimum points $\check{\xi}_{i}(i=1,2, \ldots,+\infty)$ on $\xi$-axis, such that

$$
\begin{gathered}
-\infty<\cdots<\check{\xi}_{n}<\widehat{\xi}_{n}<\cdots<\check{\xi}_{1}<\widehat{\xi}_{1}<+\infty, \\
\lim _{n \rightarrow \infty} \widehat{\xi}_{n}=\lim _{n \rightarrow \infty} \check{\xi}_{n}=-\infty, \\
U(+\infty)<u\left(\check{\xi}_{1}\right)<\cdots<U\left(\check{\xi}_{n}\right)<\cdots<U(-\infty) \\
<\cdots<U\left(\widehat{\xi}_{n}\right)<\cdots<U\left(\widehat{\xi}_{1}\right), \\
\lim _{n \rightarrow \infty} U\left(\widehat{\xi}_{n}\right)=\lim _{n \rightarrow \infty} U\left(\check{\xi}_{n}\right)=U(-\infty),
\end{gathered}
$$

and (21) holds.

Proof. (i) By using the theory of planar dynamical systems, it is obtained that $P_{1_{-}}$is an unstable focus and $P_{0}$ is a saddle point. The orbit $L\left(P_{1_{-}}, 0\right)$ tends to $P_{1_{-}}$spirally as $\xi \rightarrow-\infty$. The intersection points of $L\left(P_{1_{-}}, 0\right)$ and $\phi$ axis at the right hand of $P_{1}$ correspond to the maximum points of $U(\xi)$, while the ones at the left hand of $P_{1}$ correspond to the minimum points of $U(\xi)$. Hence, (20) hold. When $L\left(P_{1_{-}}, 0\right)$ approaches to $P_{1_{-}}$sufficiently, its properties tend 
to the properties of linear approximate solution of (8) at $P_{1_{-}}$. The frequency of $L\left(P_{1_{-}}, 0\right)$ rotating around $P_{1_{-}}$tends to $\sqrt{\left(4 \beta\left(b \phi_{1}^{2}+a \phi_{1}-c\right)-r^{2}\right)} / 4 \pi \beta$. Therefore, (21) holds.

(ii) The proof is similar to that of (i).

\section{Approximate Damped Oscillatory Solutions}

4.1. Preliminary Work. In order to obtain approximate damped oscillatory solutions, it is necessary to understand the behaviors of bounded orbits of the planar dynamical system corresponding to (1). By the bifurcation theory of planar dynamical systems, Tang et al. [6] and Liu and Li [7] have investigated (1) under various parameters conditions, respectively. Combining the conclusions associated with $g=$ $0, a \neq 0, b \geq 0$, which were obtained by Tang et al. [6] and Liu and Li [7], with the analysis of types of singular points at infinity, the global phase portraits can be obtained. For clarity and nonrepetitiveness, we only present the global phase portraits in the case $a<0$.

(i) $p$ is an even number (see Figures 10-12).

(ii) $p$ is an odd number (see Figures 13-16).

Remark 17. (i) $P_{0}, P_{i_{+}}(i=1,2,3,4), A_{i}(i=1,2)$ in Figures $10,11,12,13,14,15$, and 16 have the same meaning as those in Figures 1-9. (ii) It is easily seen that when $\beta>0, p$ is an even number, $a>0, b \geq 0$, and $c>0$, the global phase portrait is similar to that shown in Figure 10, and when $p$ is an odd number, the global phase portraits with $a>0$ are similar to those with $a<0$ shown in Figures 13-16.

By the integral method, Tang et al. [6] and Liu and Li [7] presented some bell profile solitary wave solutions. These solutions are equivalent to those obtained by Zhang et al. [8].

Theorem 18. Suppose that $\beta>0, b>0$, and $c>0$.

(i) When $p$ is an even number, there exist two bell profile solitary wave solutions in the form of $U_{1}^{ \pm}(\xi)= \pm \sqrt[p]{\varphi_{1}(\xi)}$ for (1), where

$\varphi_{1}(\xi)$

$$
\begin{gathered}
=\left((p+1)(p+2) c \sqrt{\frac{2 p+1}{a^{2}(2 p+1)+b c(p+1)(p+2)^{2}}}\right. \\
\left.\cdot \operatorname{sech}^{2}\left(\frac{p}{2} \sqrt{\frac{c}{\beta}}\left(\xi-\xi_{0}\right)\right)\right) \\
\cdot\left(2+\left(-1+a \sqrt{\frac{2 p+1}{a^{2}(2 p+1)+b c(p+1)(p+2)^{2}}}\right)\right. \\
\left.\cdot \operatorname{sech}^{2}\left(\frac{p}{2} \sqrt{\frac{c}{\beta}}\left(\xi-\xi_{0}\right)\right)\right)^{-1} \cdot
\end{gathered}
$$

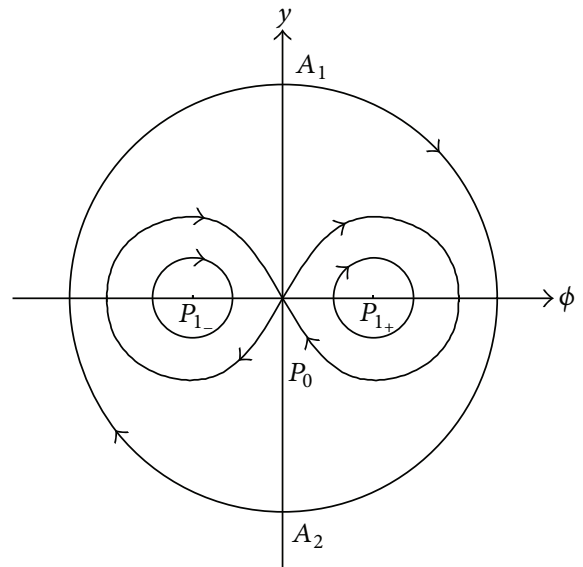

Figure 10: $(\beta>0, a<0, b>0, c>0)$.

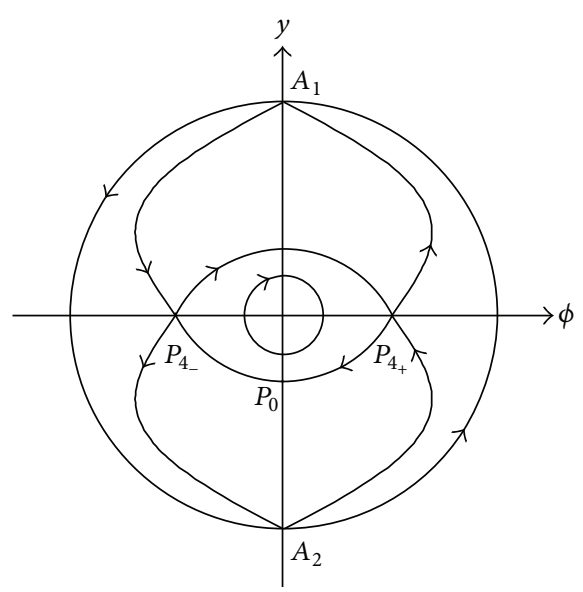

Figure 11: $(\beta>0, a<0, b=0, c<0)$.

(ii) When $p$ is an odd number, there exist two bell profile solitary wave solutions in the form of $U_{i}(\xi)=$ $\sqrt[p]{\varphi_{i}(\xi)}(i=1,2)$ for $(1)$, where $\varphi_{1}(\xi)$ is given by (23), and

$\varphi_{2}(\xi)$

$$
\begin{gathered}
=\left(-(p+1)(p+2) c \sqrt{\frac{2 p+1}{a^{2}(2 p+1)+b c(p+1)(p+2)^{2}}}\right. \\
\left.\cdot \operatorname{sech}^{2}\left(\frac{p}{2} \sqrt{\frac{c}{\beta}}\left(\xi-\xi_{0}\right)\right)\right) \\
\cdot\left(2+\left(-1-a \sqrt{\frac{2 p+1}{a^{2}(2 p+1)+b c(p+1)(p+2)^{2}}}\right)\right. \\
\left.\cdot \operatorname{sech}^{2}\left(\frac{p}{2} \sqrt{\frac{c}{\beta}}\left(\xi-\xi_{0}\right)\right)\right)^{-1} .
\end{gathered}
$$




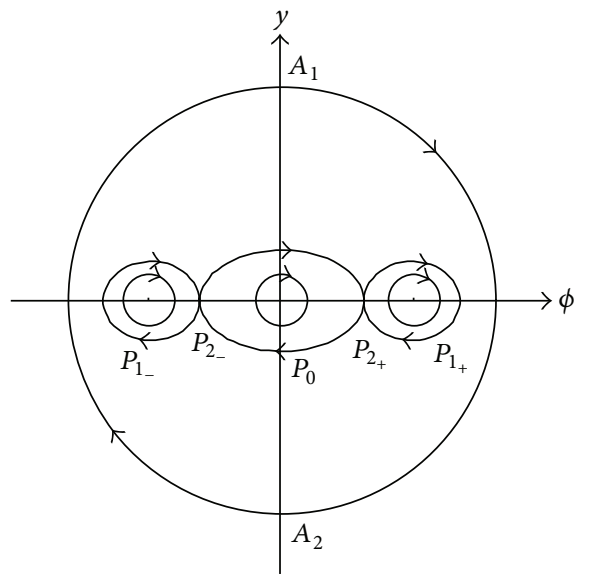

(a) $(\Delta>0)$

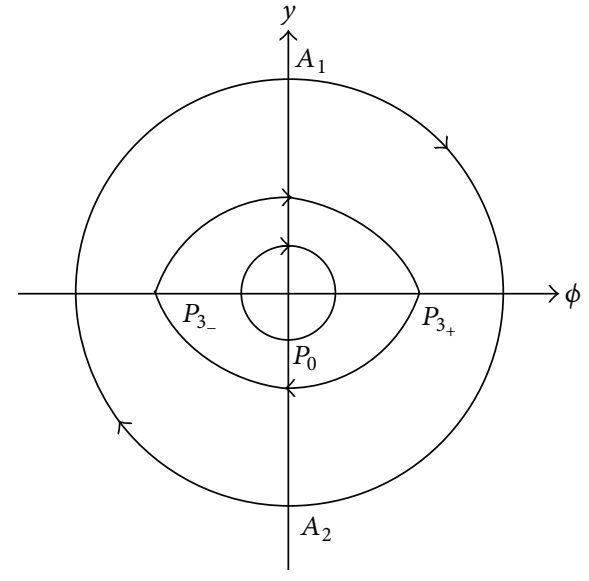

(b) $(\Delta=0)$

FIGURE 12: $(\beta>0, a<0, b>0, c<0)$.

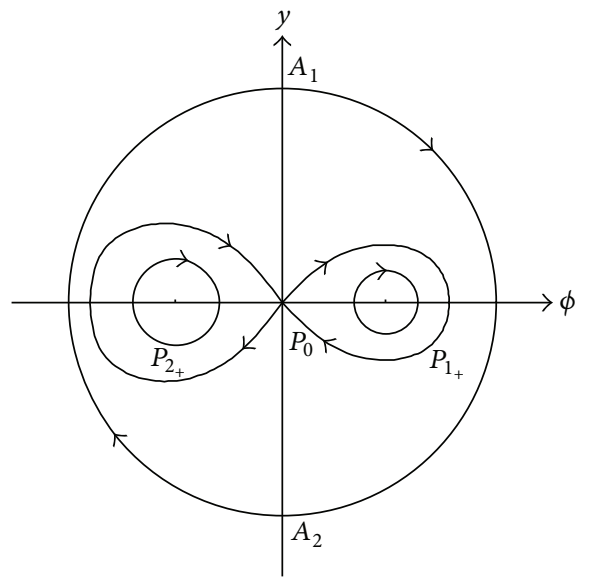

Figure 13: $(\beta>0, a<0, b>0, c>0)$.

Theorem 19. Suppose that $\beta>0, a>0, b=0$, and $c>0$.

(i) When $p$ is an even number, there exist two bell profile solitary wave solutions in the form of $U_{1}^{ \pm}(\xi)= \pm \sqrt[p]{\varphi_{1}(\xi)}$ for (1), where $\varphi_{1}(\xi)$ is given by (23).

(ii) When $p$ is an odd number, there exists a bell profile solitary wave solution in the form of $U_{1}(\xi)=\sqrt[p]{\varphi_{1}(\xi)}$ for (1), where $\varphi_{1}(\xi)$ is given by (23).

Theorem 20. Suppose that $\beta>0, p$ is an odd number, $a<0$, $b=0$, and $c>0$; then there exists a bell profile solitary wave solution in the form of $U_{2}(\xi)=\sqrt[p]{\varphi_{2}(\xi)}$ for $(1)$, where $\varphi_{2}(\xi)$ is given by (24).

When $a<0$, it is easy to prove that the bell profile solitary wave solutions $U_{1}^{ \pm}(\xi), U_{i}(\xi)(i=1,2), U_{2}(\xi)$ in Theorems 18 and 20 correspond to the homoclinic orbits $L\left(P_{0}, P_{0}\right)$ in
Figures 10, 13, and 15, respectively. Since the global phase portraits as $a>0$ are similar to those as $a<0$, when $a>0$, the bell profile solitary wave solutions given in Theorems 18 and 19 also correspond to the homoclinic orbits $L\left(P_{0}, P_{0}\right)$.

In addition to bell profile solitary wave solutions, Zhang et al. [8] also presented the kink profile solitary wave solution of compound $\mathrm{KdV}$-Burgers-type equation with nonlinear terms of any order. For example, the kink profile solitary wave solution (5.8) obtained by Zhang et al. [8]. Let $k, m$, and $\delta$ equal to $a, p$, and $\beta$, respectively, we have the kink profile solitary wave solution with the wave speed $c=-2 r^{2}(p+$ $2) / \beta(p+4)^{2}$ for $(2)$; that is, $U_{3}(\xi)=\sqrt[p]{\varphi_{3}(\xi)}$, where

$$
\begin{aligned}
\varphi_{3}(\xi) & \\
=- & \frac{r^{2}(p+1)(p+2)}{a \beta(p+4)^{2}} \\
& \cdot\left(1-\tanh \left(\frac{p r}{2 \beta(p+4)}\left(\xi-\xi_{0}\right)\right)\right. \\
& \left.-\frac{1}{2} \operatorname{sech}^{2}\left(\frac{p r}{2 \beta(p+4)}\left(\xi-\xi_{0}\right)\right)\right) .
\end{aligned}
$$

It can be proved that when $p$ is an even number, the kink profile solitary wave solutions $\pm U_{3}(\xi)$ correspond to the heteroclinic orbits $L\left(P_{0}, P_{4_{+}}\right)$in Figure 2(a), and when $p$ is an odd number, the kink profile solitary wave solution $U_{3}(\xi)$ corresponds to the heteroclinic orbit $L\left(P_{0}, P_{4_{+}}\right)$in Figure 8(a).

4.2. Approximate Damped Oscillatory Solutions of (2). By the theory of rotated vector field, it is clear that (i) the focus-saddle orbits $L\left(P_{1_{+}}, P_{0}\right)$ in Figure 1(b) and the focussaddle orbit $L\left(P_{1_{+}}, P_{0}\right)$ in Figures 5(b) and 5(c) are generated from the break of the homoclinic orbits $L\left(P_{0}, P_{0}\right)$ in 


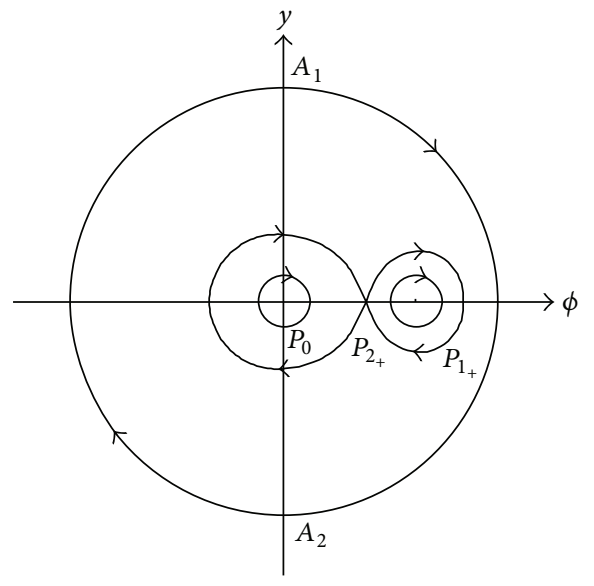

(a) $(\Delta>0)$

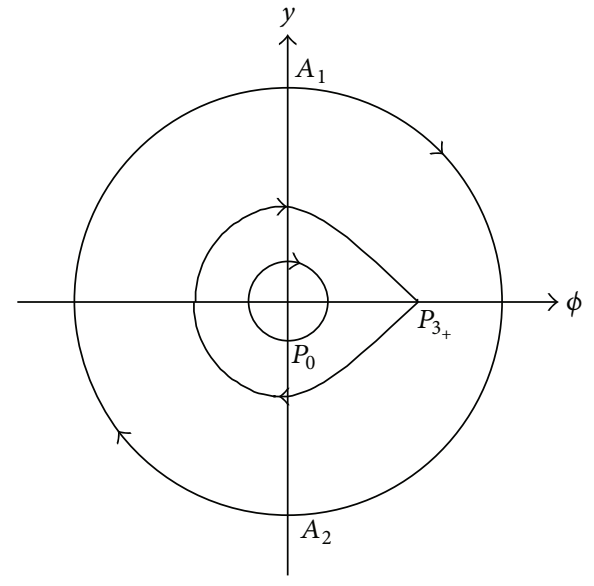

(b) $(\Delta=0)$

FiguRE 14: $(\beta>0, a<0, b>0, c<0)$.

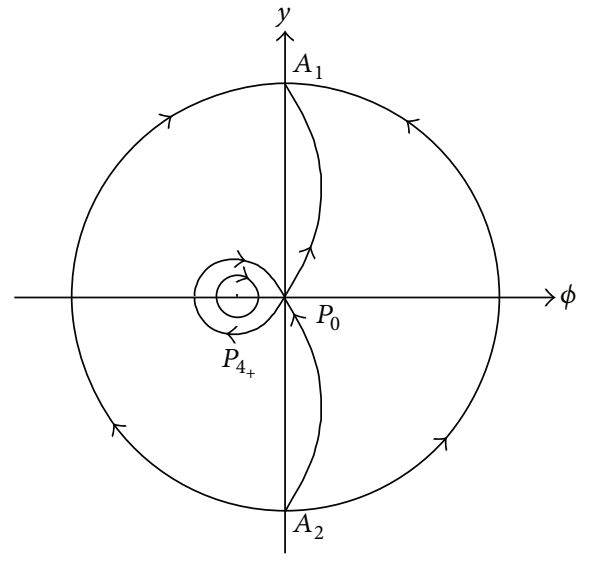

Figure 15: $(\beta>0, a<0, b=0, c>0)$.

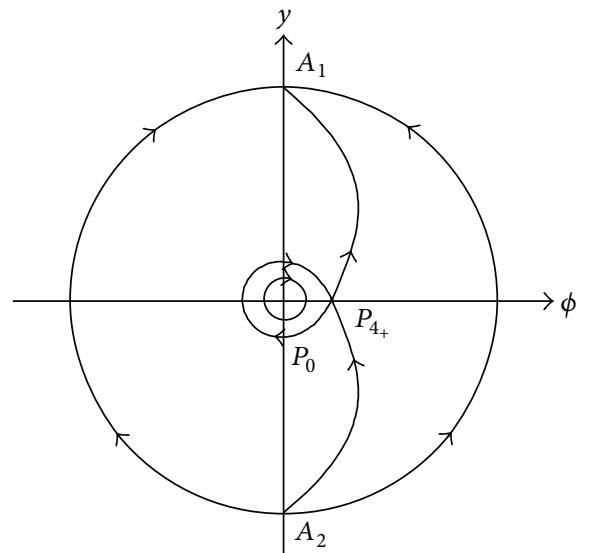

Figure 16: $(\beta>0, a<0, b=0, c<0)$.
Figure 10 and the right homoclinic orbit $L\left(P_{0}, P_{0}\right)$ in Figure 13 under the effect of the dissipation term $r U_{x x}(\xi)$, that is, $-\sqrt{4 b p \beta \phi_{1}\left(\phi_{1}-\phi_{2}\right) /(2 p+1)}<r<0$, (ii) the focussaddle orbit $L\left(P_{2}, P_{0}\right)$ in Figure 5(c) is generated from the break of the left homoclinic orbit $L\left(P_{0}, P_{0}\right)$ in Figure 13 as $-\sqrt{4 b p \beta \phi_{2}\left(\phi_{2}-\phi_{1}\right) /(2 p+1)}<r<0$, and (iii) the focus-saddle orbit $L\left(P_{4_{+}}, P_{0}\right)$ in Figure $9(\mathrm{~b})$ is generated from the break of the homoclinic orbit $L\left(P_{0}, P_{0}\right)$ in Figure 15 as $-\sqrt{4 p \beta c}<r<0$. We take the focus-saddle orbit $L\left(P_{1_{+}}, P_{0}\right)$ in Figure $1(\mathrm{~b})$ as an example to construct an approximate damped oscillatory solution corresponding to it. Other approximate damped oscillatory solutions can be constructed similarly.

Since the focus-saddle orbit $L\left(P_{1_{+}}, P_{0}\right)$ in Figure $1(\mathrm{~b})$ is generated from the break of the right homoclinic orbit $L\left(P_{0}, P_{0}\right)$ in Figure 10, the nonoscillatory part of the damped oscillatory solution corresponding to the orbit $L\left(P_{1_{+}}, P_{0}\right)$ can be expressed by the bell profile solitary wave solution

$$
U^{*}(\xi)=\sqrt[p]{\varphi_{1}(\xi)}, \quad \xi \in\left[\xi_{0},+\infty\right),
$$

where $\varphi_{1}(\xi)$ is given by (23), while the oscillatory part of the damped oscillatory wave solution can be approximated by

$$
\begin{array}{r}
U(\xi) \\
=e^{\alpha\left(\xi-\xi_{0}\right)}\left(A_{1} \cos \left(B\left(\xi-\xi_{0}\right)\right)-A_{2} \sin \left(B\left(\xi-\xi_{0}\right)\right)\right)+C, \\
\xi \in\left(-\infty, \xi_{0}\right),
\end{array}
$$

where $A_{1}, A_{2}, B, C$, and $\alpha$ are arbitrary constants which will be determined later. (27) has both damped and oscillatory properties, due to the fact that $e^{\alpha\left(\xi-\xi_{0}\right)}$ has damped property and $A_{1} \cos \left(B\left(\xi-\xi_{0}\right)\right)-A_{2} \sin \left(B\left(\xi-\xi_{0}\right)\right)$ has oscillatory property. 
Substituting (27) into (7) and omitting the terms including $e^{\alpha\left(\xi-\xi_{0}\right)}$, it is obtained that

$$
\begin{aligned}
& B^{2}=\frac{-r^{2}+4 \beta\left(b C^{2 p}+a C^{p}-c\right)}{4 \beta^{2}}, \\
& \alpha=-\frac{r}{2 \beta}, \\
& \frac{b}{2 p+1} C^{2 p}+\frac{a}{p+1} C^{p}-c=0 .
\end{aligned}
$$

To obtain approximate damped oscillatory solution to (2), some conditions to connect (26) and (27) are needed. The properties of traveling wave solutions keep the same as translating on $\xi$-axis; therefore, $\xi_{0}=0$, and

$$
\frac{d^{i}}{d \xi^{i}} U(0)=\frac{d^{i}}{d \xi^{i}} U^{*}(0), \quad i=0,1 ;
$$

that is,

$$
\begin{aligned}
A_{1}+C & =U^{*}(0), \\
\alpha A_{1}-A_{2} B & =0
\end{aligned}
$$

can be chosen as a connective point and connective conditions, respectively.

Since (27) tends to $\sqrt[p]{\phi_{1}}$ as $\xi \rightarrow-\infty, C=\sqrt[p]{\phi_{1}}$. Furthermore, from (28) and (30), it is obtained that

$$
\begin{aligned}
& B^{2}=\frac{-r^{2}(2 p+1)+4 b p \beta \phi_{1}\left(\phi_{1}-\phi_{2}\right)}{4 \beta^{2}(2 p+1)}, \\
& A_{1}=\sqrt[p]{\varphi_{1}(0)}-\sqrt[p]{\phi_{1}}, \\
& A_{2}=\frac{\alpha A_{1}}{B} .
\end{aligned}
$$

No matter what $B$ takes in (31), the value of $A_{1} \cos (B \xi)-$ $A_{2} \sin (B \xi)$ is always the same. Without loss of generality, it is assumed that $B>0$ throughout the remainder of this paper.

Summarizing the above analysis, we have the following theorems.

Theorem 21. Suppose that $\beta>0, a<0, b>0, c>0$, $-\sqrt{4 b p \beta \phi_{1}\left(\phi_{1}-\phi_{2}\right) /(2 p+1)}<r<0$.

(i) When $p$ is an even number, (2) has two damped oscillatory solutions. The approximate solution of the one corresponding to the focus-saddle orbit $L\left(P_{1_{+}}, P_{0}\right)$ in Figure $1(b)$ is

$$
\begin{aligned}
& U(\xi) \\
& \approx \begin{cases}\sqrt[p]{\varphi_{1}(\xi)}, & \xi \in[0,+\infty), \\
e^{-(r / 2 \beta) \xi}\left(A_{1} \cos (B \xi)-A_{2} \sin (B \xi)\right) & \\
+\sqrt[p]{\phi_{1}}, & \xi \in(-\infty, 0),\end{cases}
\end{aligned}
$$

where $\varphi_{1}(\xi), B, A_{1}$, and $A_{2}$ are given by (23), (31), and (32), respectively. The approximate solution of the one corresponding to the focus-saddle orbit $L\left(P_{1_{-}}, P_{0}\right)$ in Figure 1(b) is

$$
\begin{aligned}
& U(\xi) \\
& \approx \begin{cases}-\sqrt[p]{\varphi_{1}(\xi)}, & \xi \in[0,+\infty), \\
e^{-(r / 2 \beta) \xi}\left(A_{1} \cos (B \xi)-A_{2} \sin (B \xi)\right) & \\
-\sqrt[p]{\phi_{1}}, & \xi \in(-\infty, 0),\end{cases}
\end{aligned}
$$

where $\varphi_{1}(\xi)$ and $B$ are given by (23) and (31), respectively, $A_{1}=-\sqrt[p]{\varphi_{1}(0)}+\sqrt[p]{\phi_{1}}, A_{2}=\alpha A_{1} / B$.

(ii) When $p$ is an odd number, (2) has a damped oscillatory solution corresponding to the focus-saddle orbit $L\left(P_{1_{+}}, P_{0}\right)$ in Figures $5(b)$ and $2(c)$, whose approximate solution is (33).

Theorem 22. When $\beta>0, p$ is an odd number, $a<0$, $b>0, c>0,-\sqrt{4 b p \beta \phi_{2}\left(\phi_{2}-\phi_{1}\right) /(2 p+1)}<r<0$, (2) has a damped oscillatory solution corresponding to the focus-saddle orbit $L\left(P_{2_{+}}, P_{0}\right)$ in Figure 5(c), whose approximate solution is

$$
\begin{aligned}
& U(\xi) \\
& \approx \begin{cases}\sqrt[p]{\varphi_{2}(\xi)}, & \xi \in[0,+\infty), \\
e^{-(r / 2 \beta) \xi}\left(A_{1} \cos (B \xi)-A_{2} \sin (B \xi)\right) & \\
+\sqrt[p]{\phi_{2}}, & \xi \in(-\infty, 0),\end{cases}
\end{aligned}
$$

where $\varphi_{2}(\xi)$ is given by (24), $B=(1 / 2 \beta) \times$ $\sqrt{-r^{2}+4 b p \beta \phi_{2}\left(\phi_{2}-\phi_{1}\right) /(2 p+1)}, A_{1}=\sqrt[p]{\varphi_{2}(0)}-\sqrt[p]{\phi_{2}}$, and $A_{2}=\alpha A_{1} / B$.

Theorem 23. When $\beta>0, p$ is an odd number, $a<0, b=0$, $c>0,-\sqrt{4 p \beta c}<r<0$, (2) has a damped oscillatory wave solution corresponding to the focus-saddle orbit $L\left(P_{4_{+}}, P_{0}\right)$ in Figure 9(b), whose approximate solution is

$$
\begin{aligned}
& U(\xi) \\
& \approx \begin{cases}\sqrt[p]{\varphi_{2}(\xi),} & \xi \in[0,+\infty), \\
e^{-(r / 2 \beta) \xi}\left(A_{1} \cos (B \xi)-A_{2} \sin (B \xi)\right) & \\
+\sqrt[p]{\phi_{4}}, & \xi \in(-\infty, 0),\end{cases}
\end{aligned}
$$

where $\varphi_{2}(\xi)$ is given by (24), $B=(1 / 2 \beta) \sqrt{-r^{2}+4 p \beta c}, A_{1}=$ $\sqrt[p]{\varphi_{2}(0)}-\sqrt[p]{\phi_{4}}$, and $A_{2}=\alpha A_{1} / B$.

Since the global phase portraits with $a>0$ are similar to those with $a<0$, Theorems 21 and 22 are also established as $a>0$, and for the case $\beta>0, a>0, b=0, c>0,-\sqrt{4 p \beta c}<$ $r<0$, we have the following theorem. 
Theorem 24. Suppose that $\beta>0, a>0, b=0, c>0$, $-\sqrt{4 p \beta c}<r<0$.

(i) When $p$ is an even number, (2) has two damped oscillatory solutions, whose approximate solutions are

$$
\begin{aligned}
& U(\xi) \\
& \approx \begin{cases}\sqrt[p]{\varphi_{1}(\xi)}, & \xi \in[0,+\infty), \\
e^{-(r / 2 \beta) \xi}\left(A_{1} \cos (B \xi)-A_{2} \sin (B \xi)\right) & \\
+\sqrt[p]{\phi_{4}}, & \xi \in(-\infty, 0),\end{cases}
\end{aligned}
$$

where $\varphi_{1}(\xi)$ is given by (23), $B=(1 / 2 \beta) \sqrt{-r^{2}+4 p \beta c}$, $A_{1}=\sqrt[p]{\varphi_{1}(0)}-\sqrt[p]{\phi_{4}}, A_{2}=\alpha A_{1} / B$, and

$U(\xi)$

$$
\approx \begin{cases}-\sqrt[p]{\varphi_{1}(\xi)}, & \xi \in[0,+\infty), \\ e^{-(r / 2 \beta) \xi}\left(A_{1} \cos (B \xi)-A_{2} \sin (B \xi)\right) & \\ -\sqrt[p]{\phi_{4}}, & \xi \in(-\infty, 0),\end{cases}
$$

where $\varphi_{1}(\xi)$ is given by (23), $B=(1 / 2 \beta) \sqrt{-r^{2}+4 p \beta c}$, $A_{1}=-\sqrt[p]{\varphi_{1}(0)}+\sqrt[p]{\phi_{4}}$, and $A_{2}=\alpha A_{1} / B$, respectively.

(ii) When $p$ is an odd number, (2) has a damped oscillatory solution, whose approximate solution is (37).

With the help of MATLAB 7.0, the approximate damped oscillatory solutions (33) and (34) are plotted with $a=-0.2$, $b=0.6, c=0.2, p=2, \beta=1$, and $r=-0.5$ in the intervals $x \in[-60,40]$ and $t \in[0,1]$, respectively (see Figures 17 and 18). The graphics of approximate damped oscillatory solutions (35)-(37) are similar to that shown in Figure 18, and the graphic of approximate damped oscillatory solution (38) is similar to that shown in Figure 17.

\subsection{Effect of Parameters on Frequency and Period of Approxi-} mate Damped Oscillatory Solutions. In this section, we aim to analyze effect of parameters $r, \beta, a, b, c, p$ on frequency $f$ and period $T$ of approximate damped oscillatory solution (33). For other approximate damped oscillatory solutions (34)(38), we can obtain similar conclusions.

The approximate damped oscillatory solution (33) in the interval $\xi \in(-\infty, 0)$ can be rewritten as

$$
U(\xi) \approx e^{-(r / 2 \beta) \xi} A_{3} \cos (B \xi+\varphi)+\sqrt[p]{\phi_{1}},
$$

where $B, A_{1}, A_{2}$ are given by (31), (32), $A_{3}=\sqrt{A_{1}^{2}+A_{2}^{2}}, \varphi=$ $\tan ^{-1}\left(A_{2} / A_{1}\right)$. Equation (39) indicates that the variation law of $U(\xi)$ approximates to that of simple harmonic vibration. It is easily obtained that the frequency and period of $U(\xi)$ approximate to $f=B / 2 \pi$ and $T=2 \pi / B$. According to

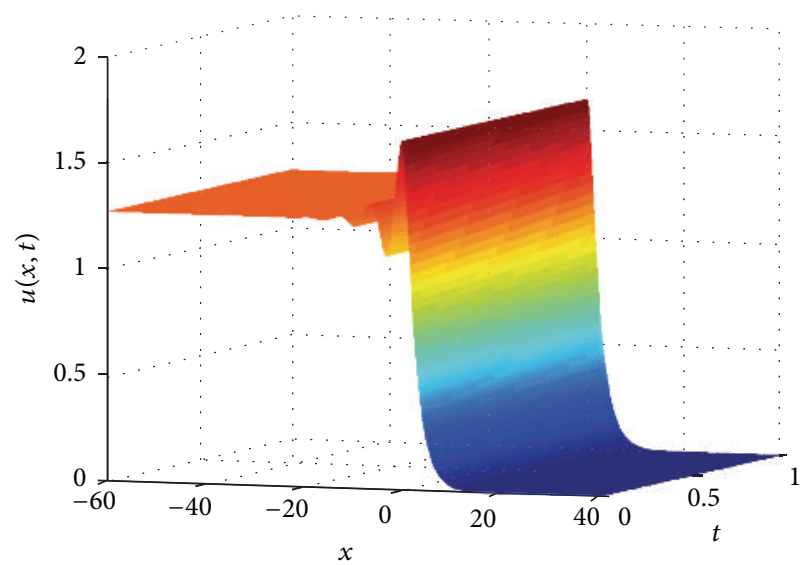

FIGURE 17: Plot of the approximate damped oscillatory solution (33) with $a=-0.2, b=0.6, c=0.2, p=2, \beta=1$, and $r=-0.5$.

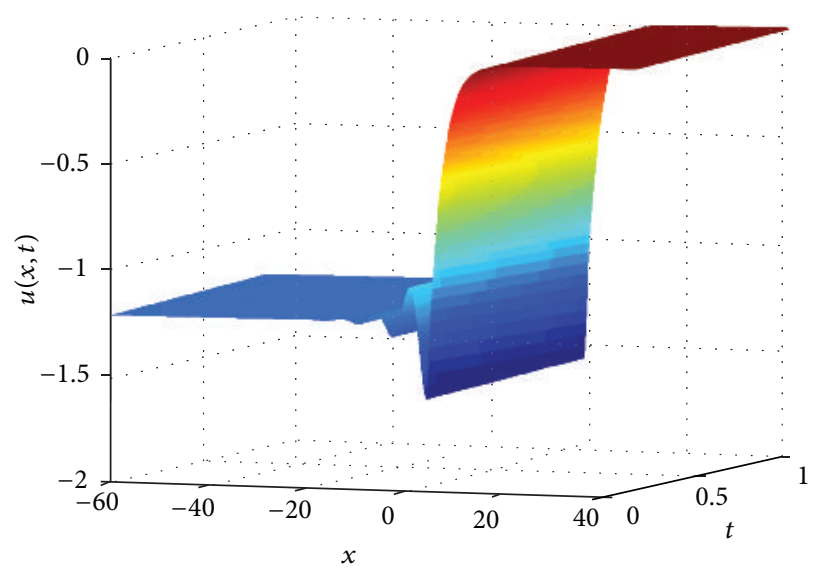

FIGURE 18: Plot of the approximate damped oscillatory solution (34) with $a=-0.2, b=0.6, c=0.2, p=2, \beta=1$, and $r=-0.5$.

the relation between frequency $f$ and period $T$, we only give effect of parameters $r, \beta, a, b, c$, and $p$ on period $T$. The results on effect of parameters $r, \beta, a, b, c$, and $p$ on frequency $f$ are opposite to those on period $T$.

4.3.1. Effect of $r, \beta$ on Period T. For given values of $a, b$, $c$, and $p$, the value of $-4 b p \phi_{1}\left(\phi_{1}-\phi_{2}\right) /(2 p+1)$ is fixed accordingly. Furthermore, it is concluded that (i) for any fixed $\beta, T$ is increasing as $r$ decreases, (ii) for any fixed $r$, when $\beta>2 r^{2}(2 p+1) / 4 b p \phi_{1}\left(\phi_{1}-\phi_{2}\right), T$ is increasing as $\beta$ increases, and when $\beta<2 r^{2}(2 p+1) / 4 b p \phi_{1}\left(\phi_{1}-\phi_{2}\right), T$ is decreasing as $\beta$ increases. Figure 19 displays the relation between $r, \beta$, and $T$.

4.3.2. Effect of $a, b, c$, and $p$ on Period T. For any fixed $r, \beta$, it can be proved that as $\sqrt{4 b p \phi_{1}\left(\phi_{1}-\phi_{2}\right) /(2 p+1)}$ increases, $T$ is decreasing. Since the value of $\sqrt{4 b p \phi_{1}\left(\phi_{1}-\phi_{2}\right) /(2 p+1)}$ is proportional to the value of $p$ or $c$ and is inversely proportional to the value of $a$ or $b, T$ is decreasing as $p$ or $c$ 


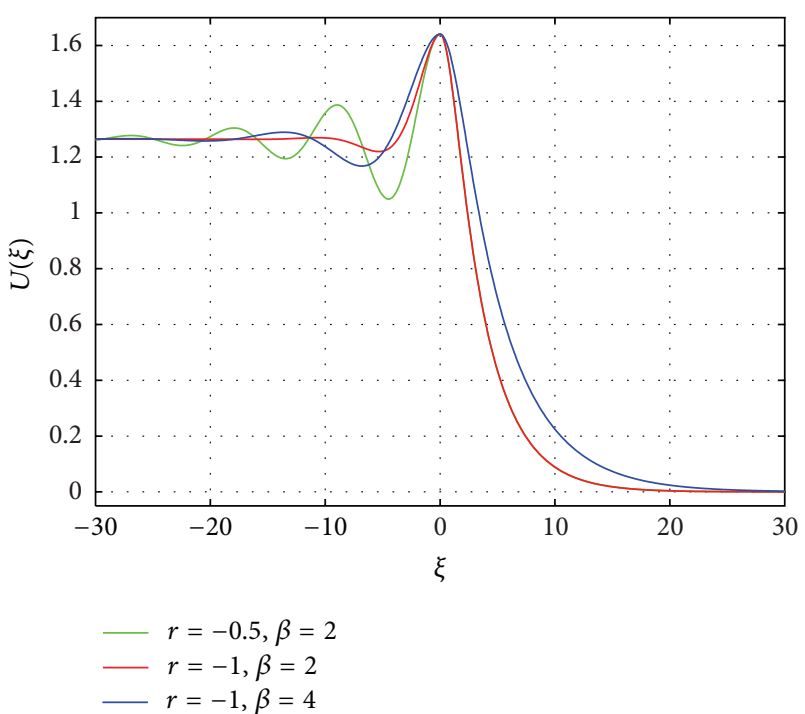

FIGURE 19: Plot of the approximate damped oscillatory solution (33) for different values of $r$, $\beta$ with $a=-0.2, b=0.6, c=0.2$, and $p=2$.

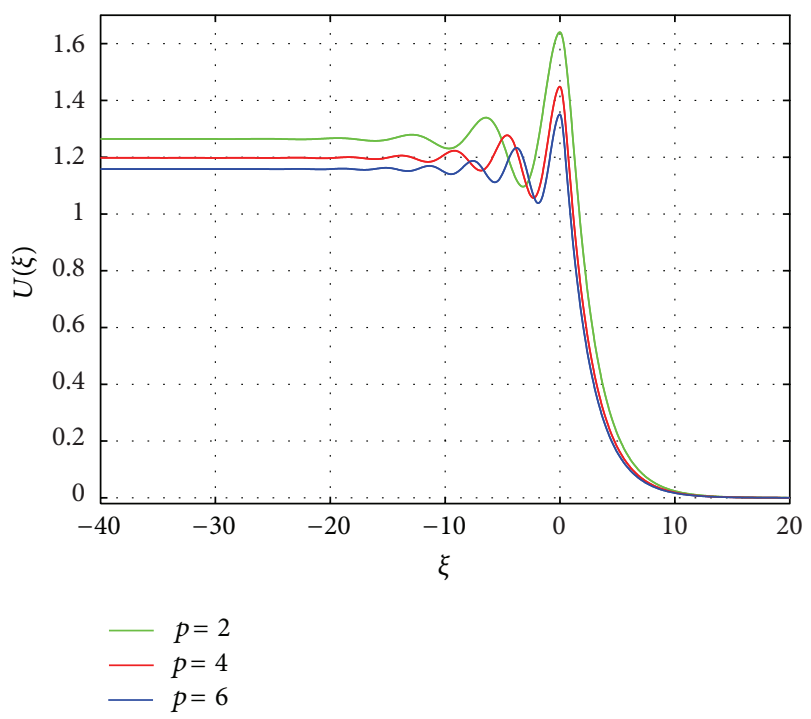

FIGURE 20: Plot of the approximate damped oscillatory solution (33) for different value of $p$ with $a=-0.2, b=0.6, c=0.2, \beta=1$, and $r=-1$.

increases, and it is increasing as $a$ or $b$ increases. The graphics of (33) for different values of $p, c, a$, or $b$ are shown in Figures 20,21 , and 22 , respectively.

\section{Error Estimates and Discussion}

In this section, we first study error estimate of the approximate damped oscillatory solution (33) and then make some discussion on the exact damped oscillatory solution corresponding to (33). Similar results can be obtained for other approximate damped oscillatory solutions and exact damped oscillatory solutions corresponding to them.

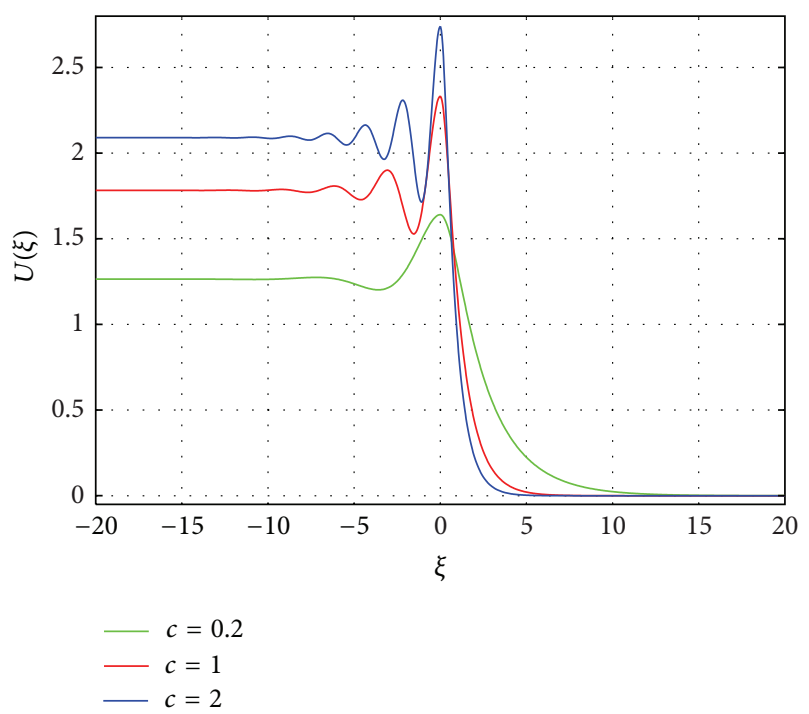

FIGURE 21: Plot of the approximate damped oscillatory solution (33) for different value of $c$ with $a=-0.2, b=0.6, p=2, \beta=1$, and $r=-1$.

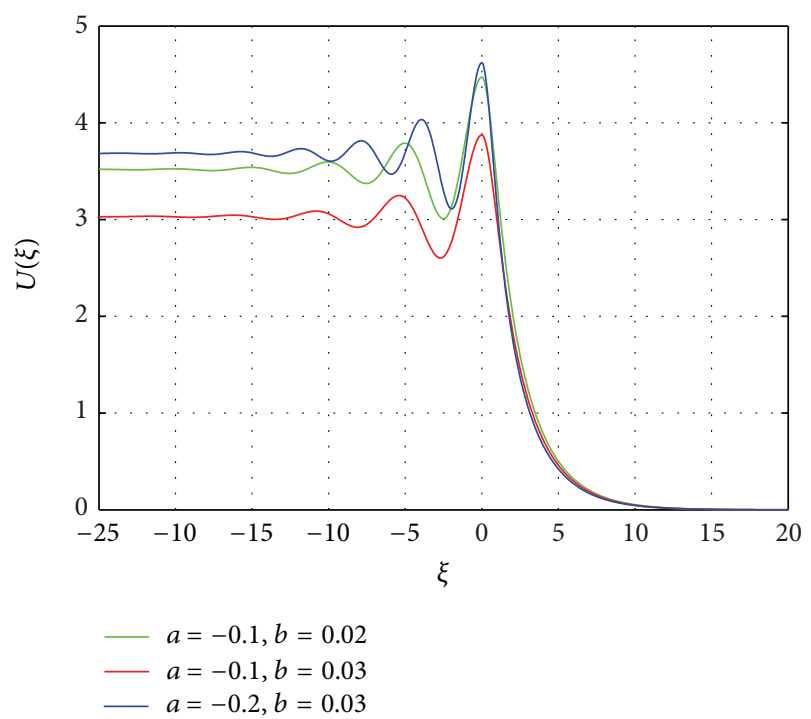

FIGURE 22: Plot of the approximate damped oscillatory solution (33) for different values of $a, b$ when $c=0.2, p=2, \beta=1$, and $r=-0.5$.

5.1. Error Estimates of Approximate Damped Oscillatory Solutions. Substituting

$$
V(\xi)=\frac{U(\xi)-\sqrt[p]{\phi_{1}}}{-2 \sqrt[p]{\phi_{1}}}
$$

and $\xi=-\eta(\eta>0)$ into (7), the problem of finding an exact damped oscillatory solution of (7), which is subject to

$$
\begin{aligned}
U(0) & =\sqrt[p]{\varphi_{1}(0)}, \\
U^{\prime}(0) & =0,
\end{aligned}
$$


is converted into solving the following initial value problem:

$$
\begin{gathered}
\bar{V}_{\eta \eta}(\eta)-\frac{r}{\beta} \bar{V}_{\eta}(\eta)+\frac{b}{(2 p+1) \beta} \\
\cdot\left(\bar{V}(\eta)-\frac{1}{2}\right) \bar{f}(\eta) \bar{g}(\eta)=0, \\
\bar{V}(0)=\frac{\sqrt[p]{\varphi_{1}(0)}-\sqrt[p]{\phi_{1}}}{-2 \sqrt[p]{\phi_{1}}} \\
\bar{V}_{\eta}(0)=0
\end{gathered}
$$

where $\bar{V}(\eta)=V(\xi), \bar{f}(\eta)=2^{p} \phi_{1}(V(\xi)-1 / 2)^{p}-\phi_{1}$, and $\bar{g}(\eta)=$ $2^{p} \phi_{1}(V(\xi)-(1 / 2))^{p}-\phi_{2}$. Simplifying the above initial value problem yields

$$
\begin{gathered}
\bar{V}_{\eta \eta}(\eta)-\frac{r}{\beta} \bar{V}_{\eta}(\eta)+\frac{b p \phi_{1}\left(\phi_{1}-\phi_{2}\right)}{(2 p+1) \beta} \bar{V}(\eta) \\
+\frac{b \phi_{1}\left(\phi_{1}-\phi_{2}\right)}{(2 p+1) \beta} \bar{V}^{2}(\eta) \bar{h}(\eta)=0 \\
\bar{V}(0)=\frac{\sqrt[p]{\varphi_{1}(0)}-\sqrt[p]{\phi_{1}}}{-2 \sqrt[p]{\phi_{1}}} \\
\bar{V}_{\eta}(0)=0
\end{gathered}
$$

where $\bar{h}(\eta)$ satisfies

$$
\begin{gathered}
\frac{b}{(2 p+1) \beta}\left(\bar{V}(\eta)-\frac{1}{2}\right) \bar{f}(\eta) \bar{g}(\eta) \\
=\frac{b p \phi_{1}\left(\phi_{1}-\phi_{2}\right)}{(2 p+1) \beta} \bar{V}(\eta) \\
+\frac{b \phi_{1}\left(\phi_{1}-\phi_{2}\right)}{(2 p+1) \beta} \bar{V}^{2}(\eta) \bar{h}(\eta) .
\end{gathered}
$$

By the thought of homogeneous principle, we obtain the implicit exact solution of (43); that is,

$$
\begin{aligned}
& \bar{V}(\eta) \\
& =e^{\alpha_{1} \eta}\left(c_{1} \cos \left(\beta_{1} \eta\right)+c_{2} \sin \left(\beta_{1} \eta\right)\right) \\
& \quad+\frac{b \phi_{1}\left(\phi_{1}-\phi_{2}\right)}{(2 p+1) \beta \beta_{1}} \int_{0}^{\eta} e^{\alpha_{1}(\eta-\tau)} \sin \left(\beta_{1}(\eta-\tau)\right) \bar{V}^{2}(\tau) \bar{h}(\tau) d \tau,
\end{aligned}
$$

where $\alpha_{1}=r / 2 \beta, \beta_{1}=(1 / 2 \beta) \sqrt{-r^{2}+4 b p \beta \phi_{1}\left(\phi_{1}-\phi_{2}\right) /(2 p+1)}$, $c_{1}=\left(\sqrt[p]{\varphi_{1}(0)}-\sqrt[p]{\phi_{1}}\right) /-2 \sqrt[p]{\phi_{1}}$, and $c_{2}=-\alpha_{1} c_{1} / \beta_{1}$.
Substituting $\eta=-\xi$ into the above formula and making the transformations $t=-\tau$ and (40), we have

$$
\begin{aligned}
U(\xi)= & e^{-\alpha_{1} \xi}\left(\bar{c}_{1} \cos \left(\beta_{1} \xi\right)+\bar{c}_{2} \sin \left(\beta_{1} \xi\right)\right) \\
& +\sqrt[p]{\phi_{1}}+\frac{b \phi_{1}\left(\phi_{1}-\phi_{2}\right)}{(2 p+1) \beta \beta_{1}} \\
& \cdot \int_{\xi}^{0} e^{-\alpha_{1}(\xi-t)} \sin \left(\beta_{1}(\xi-t)\right)\left(U(t)-\sqrt[p]{\phi_{1}}\right)^{2} h_{1}(t) d t
\end{aligned}
$$

where $h_{1}(t)=\left(1 / 2 \sqrt[p]{\phi_{1}}\right) h(t), h(t)=\bar{h}(\tau), \bar{c}_{1}=\sqrt[p]{\varphi_{1}(0)}-\sqrt[p]{\phi_{1}}$, and $\bar{c}_{2}=\alpha_{1} \bar{c}_{1} / \beta_{1}$. Since $\beta_{1}, \bar{c}_{1}, \bar{c}_{2}$ are equal to $B, A_{1},-A_{2}$ in Theorem 21(1), respectively, the first two terms on the right hand side of (46) are the approximate damped oscillatory solution (33). Hence, (46) reflects the relation between (33) and the corresponding exact solution.

Since the damped oscillatory solution is bounded, there exists $M>0$, such that $|U(\xi)|<M$. Furthermore, from (46), it is obtained that

$$
\begin{aligned}
\mid U(\xi) & -\sqrt[p]{\phi_{1}} \mid \\
\leq & \bar{C}_{1} e^{-\alpha_{1} \xi}+\frac{b \phi_{1}\left(\phi_{1}-\phi_{2}\right) T_{2}}{(2 p+1) \beta \beta_{1}} \\
& \cdot \int_{\xi}^{0} e^{-\alpha_{1}(\xi-t)}\left|U(t)-\sqrt[p]{\phi_{1}}\right| d t, \quad \xi<0,
\end{aligned}
$$

where $\bar{C}_{1}=\left|\bar{c}_{1}\right|+\left|\bar{c}_{2}\right|, T_{1}$ is the upper bound of $\left|h_{1}(t)\right|$, and $T_{2}=\left(M+\sqrt[p]{\phi_{1}}\right) T_{1}$. By Gronwall inequality, the above formula becomes

$$
\left|U(\xi)-\sqrt[p]{\phi_{1}}\right| \leq \bar{C}_{2} e^{-\alpha_{1} \xi}, \quad \xi<0,
$$

where $\bar{C}_{2}=\bar{C}_{1} e^{-b \phi_{1}\left(\phi_{1}-\phi_{2}\right) T_{2} /(2 p+1) \beta \alpha_{1} \beta_{1}}$. Equation (48) is the estimate of amplitude. From (48), it is easily seen that $U(\xi)$ tends to $\sqrt[p]{\phi_{1}}$ as $\xi \rightarrow-\infty$.

Combining (46) with (48) yields

$$
\begin{gathered}
\left|U(\xi)-\left(e^{-\alpha_{1} \xi}\left(\bar{c}_{1} \cos \left(\beta_{1} \xi\right)+\bar{c}_{2} \sin \left(\beta_{1} \xi\right)\right)+\sqrt[p]{\phi_{1}}\right)\right| \\
\leq-\frac{b \phi_{1}\left(\phi_{1}-\phi_{2}\right) T_{1} \bar{C}_{2}^{2}}{(2 p+1) \beta \alpha_{1} \beta_{1}} e^{-2 \alpha_{1} \xi}\left(1-e^{-\alpha_{1} \xi}\right) \\
\leq-\frac{b \phi_{1}\left(\phi_{1}-\phi_{2}\right) T_{1} \bar{C}_{2}^{2}}{(2 p+1) \beta \alpha_{1} \beta_{1}} e^{-2 \alpha_{1} \xi}, \quad \xi<0 .
\end{gathered}
$$

Equation (49) reveals that error between the implicit exact damped oscillatory solution and the approximate damped oscillatory solution (33) is less than $\varepsilon(\xi)=-\left(b \phi_{1}\left(\phi_{1}-\right.\right.$ $\left.\left.\phi_{2}\right) T_{1} \bar{C}_{2}^{2} /(2 p+1) \beta \alpha_{1} \beta_{1}\right) e^{-2 \alpha_{1} \xi}$. Since the error is infinitesimal decreasing in the exponential form, (33) is meaningful to be an approximate solution to (2). 
5.2. Discussion. Equation (48) indicates that the implicit exact damped oscillatory solution $U(\xi)$ approaches to $\sqrt[p]{\phi_{1}}$ in exponential form $e^{-\alpha_{1} \xi}$. Therefore, for any fixed $\beta$, the smaller $r$ is, the faster the speed of $U(\xi)$ converging to $\sqrt[p]{\phi_{1}}$ is, and for any fixed $r$, the larger $\beta$ is, the smaller the speed of $U(\xi)$ converging to $\sqrt[p]{\phi_{1}}$ is. In addition, (48) also indicates that the implicit exact damped oscillatory solution $U(\xi)$ locates between two exponential curves $\sqrt[p]{\phi_{1}} \pm \bar{C}_{2} e^{-\alpha_{1} \xi}$ in the region of $\xi<0$, and its amplitude is less than $2 \bar{C}_{2} e^{-\alpha_{1} \xi}$. When $\xi \rightarrow$ $-\infty$, the amplitude tends to zero in the form of an exponential function. For any fixed $\beta$ or $r$, except the first peak, other peaks and troughs become more inconspicuous when $r$ or $\beta$ becomes smaller. Therefore, the wave is obviously flat when it is not far away from the origin, and the effort of damped oscillatory wave becomes weak rapidly. It is shown that the damped oscillatory solution of (2) under the conditions $\beta>$ $0, a<0, b>0, c>0,-\sqrt{4 b p \beta \phi_{1}\left(\phi_{1}-\phi_{2}\right) /(2 p+1)}<r<0$, and $p$ is an even number still has some properties of solitary wave solution.

\section{Conclusion}

In this paper, we mainly study approximate damped oscillatory solutions of compound KdV-Burgers-type equation with nonlinear terms of any order (2). Based on the qualitative analysis, we obtained the existence conditions and number of bounded traveling wave solutions (kink profile solitary wave solutions and damped oscillatory solutions). According to the evolution relations of bounded orbits in the global phase portraits, the structure of approximate damped oscillatory solutions traveling to the left is designed. And then, they are obtained by the undetermined coefficients method. In addition, we analyze effect of parameters $r, \beta, a, b, c$, and $p$ on frequency $f$ and period $T$ of approximate damped oscillatory solutions. The results reveal that (i) $T$ is decreasing as $p, c$, or $r$ increases, (ii) $T$ is increasing as $a$ or $b$ increases, (iii) when $\beta>2 r^{2}(2 p+1) / 4 b p \phi_{1}\left(\phi_{1}-\phi_{2}\right), T$ is increasing as $\beta$ increases, and when $\beta<2 r^{2}(2 p+1) / 4 b p \phi_{1}\left(\phi_{1}-\phi_{2}\right), T$ is decreasing as $\beta$ increases. By the idea of homogenization principle, error estimates of these approximate solutions are derived from the integral equations reflecting the relations between approximate solutions and exact solutions. It is shown that the errors are less than $\varepsilon(\xi)=K e^{-(\beta c / 2) \xi}$, where $K$ is a positive constant. Therefore, (33)-(38) are meaningful to be approximate damped oscillatory solutions of (2).

Wang and Yau [20-22] have employed differential transformation method and hybrid numerical method combining the differential transformation method and the finite difference method to analyze many systems, such as a gyroscope system and united gas-lubricated bearing system. These methods are widely used techniques for solving both linear and nonlinear differential equations due to their rapid convergence rate and minimal calculation error. These methods provide us a new approach to study (2); we will do this work in the future.

\section{Conflict of Interests}

The authors declare that there is no conflict of interests regarding the publication of this paper.

\section{Acknowledgments}

This project is supported by the National Natural Science Foundation of China (no. 11471215), Innovation Program of Shanghai Municipal Education Commission (no. 13ZZ118), Shanghai Leading Academic Discipline Project (no. XTKX2012), and the Hujiang Foundation of China (no. B14005).

\section{References}

[1] B. Dey, "Domain wall solutions of KdV-like equations with higher order nonlinearity," Journal of Physics A: Mathematical and General, vol. 19, no. 1, pp. L9-L12, 1986.

[2] M. Wadati, "Wave propagation in nonlinear lattice I," Journal of the Physical Society of Japan, vol. 38, no. 3, pp. 673-680, 1975.

[3] M. Wadati, "Wave propagation in nonlinear lattice II," Journal of the Physical Society of Japan, vol. 38, no. 3, pp. 681-686, 1975.

[4] B. Dey, KdV Like Equations with Domain Wall Solutions and their Hamiltonians, Solitons, Springer Series in Nonlinear Dynamics, Springer, New York, NY, USA, 1988.

[5] M. W. Coffey, "On series expansions giving closed-form solutions of Korteweg-de Vries-like equations," SIAM Journal on Applied Mathematics, vol. 50, no. 6, pp. 1580-1592, 1990.

[6] M. Y. Tang, R. Q. Wang, and Z. J. Jing, "Solitary waves and their bifurcations of $\mathrm{KdV}$ like equation with higher order nonlinearity," Science in China A: Mathematics, vol. 45, no. 10, pp. 1255-1267, 2002.

[7] Z. R. Liu and J. B. Li, "Bifurcations of solitary waves and domain wall waves for KdV-like equation with higher order nonlinearity," International Journal of Bifurcation and Chaos, vol. 12, no. 2, pp. 397-407, 2002.

[8] W. Zhang, Q. Chang, and B. Jiang, "Explicit exact solitarywave solutions for compound KdV-type and compound KdVBurgers-type equations with nonlinear terms of any order," Chaos, Solitons \& Fractals, vol. 13, no. 2, pp. 311-319, 2002.

[9] G. B. Whitham, Linear and Nonlinear Waves, Springer, New York, NY, USA, 1974.

[10] B. Li, Y. Chen, and H. Q. Zhang, "Auto-Bäcklund transformation and exact solutions for compound KDV-type and compound KDV-burgers-type equations with nonlinear terms of any order," Physics Letters A, vol. 305, no. 6, pp. 377-382, 2002.

[11] B. Li, Y. Chen, and H. Q. Zhang, "Explicit exact solutions for compound KDV-type and compound KDV-BUrgers-type equations with nonlinear terms of any order," Chaos, Solitons and Fractals, vol. 15, no. 4, pp. 647-654, 2003.

[12] Z. Yan and H. Zhang, "New explicit solitary wave solutions and periodic wave solutions for Whitham-Broer-Kaup equation in shallow water," Physics Letters A, vol. 285, no. 5-6, pp. 355-362, 2001.

[13] Z. Feng and R. Knobel, "Traveling waves to a Burgers-Korteweg-de Vries-type equation with higher-order nonlinearities," Journal of Mathematical Analysis and Applications, vol. 328, no. 2, pp. 1435-1450, 2007. 
[14] J. Li, M. Li, and J. Na, "Kink wave determined by parabola solution of a nonlinear ordinary differential equation," Applied Mathematics and Mechanics, vol. 28, no. 7, pp. 883-892, 2007.

[15] V. V. Nemytskii and V. V. Stepanov, Qualitative Theory of Differential Equations, Dover, New York, NY, USA, 1989.

[16] Z. F. Zhang, T. R. Ding, W. Z. Huang, and Z. X. Dong, Qualitative Theory of Differential Equations, vol. 101 of Translations of Mathematical Monographs, American Mathematical Society, Providence, RI, USA, 1992.

[17] D. G. Aronson and H. F. Weinberger, "Multidimensional nonlinear diffusion arising in population genetics," Advances in Mathematics, vol. 30, no. 1, pp. 33-76, 1978.

[18] P. C. Fife, Mathematical Aspects of Reacting and Diffusing Systems, vol. 28 of Lecture Notes in Biomathematics, Springer, New York, NY, USA, 1979.

[19] Q. X. Ye and Z. Y. Li, Introduction of Reaction Diffusion Equation, Science Press, Beijing, China, 1990.

[20] C.-C. Wang and H. T. Yau, "Nonlinear dynamic analysis and sliding mode control for a gyroscope system," Nonlinear Dynamics, vol. 66, no. 1-2, pp. 53-65, 2011.

[21] C.-C. Wang, "Bifurcation and nonlinear dynamic analysis of united gas-lubricated bearing system," Computers and Mathematics with Applications, vol. 64, no. 5, pp. 729-738, 2012.

[22] C.-C. Wang and H.-T. Yau, "Analysis of nonlinear dynamic behavior of atomic force microscope using differential transformation method," Acta Mechanica, vol. 198, no. 1-2, pp. 87-98, 2008. 


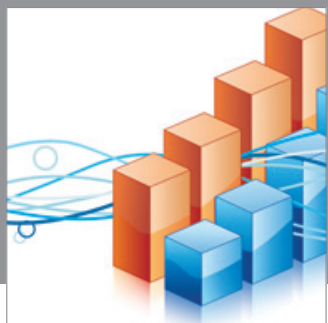

Advances in

Operations Research

mansans

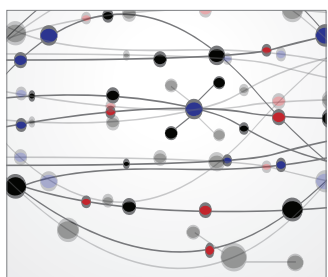

The Scientific World Journal
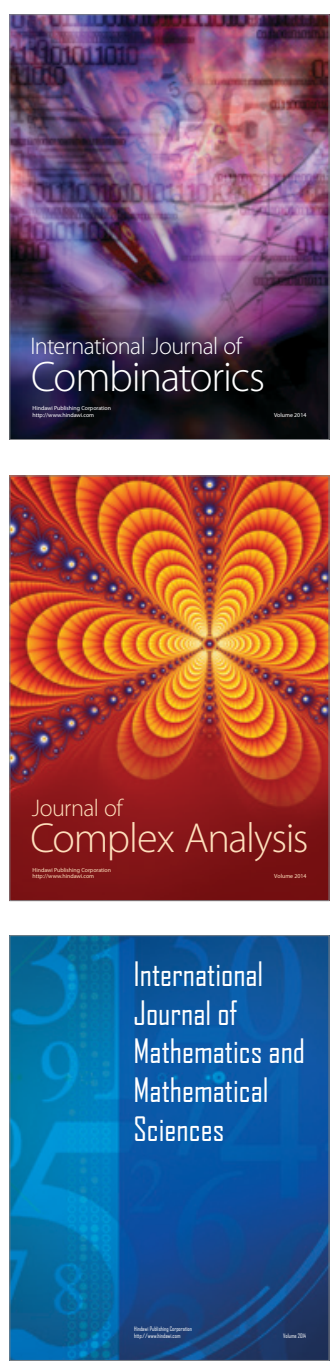
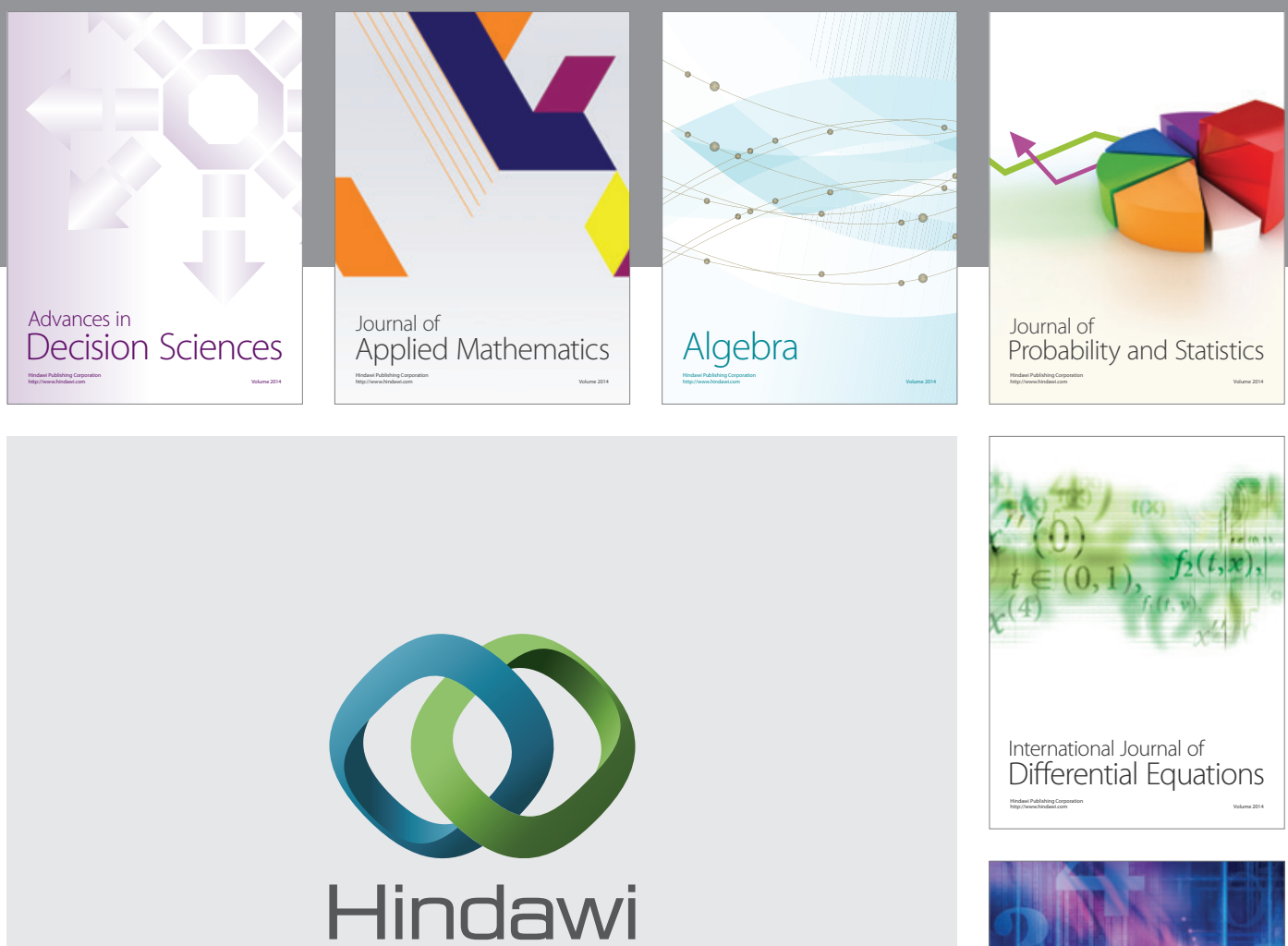

Submit your manuscripts at http://www.hindawi.com
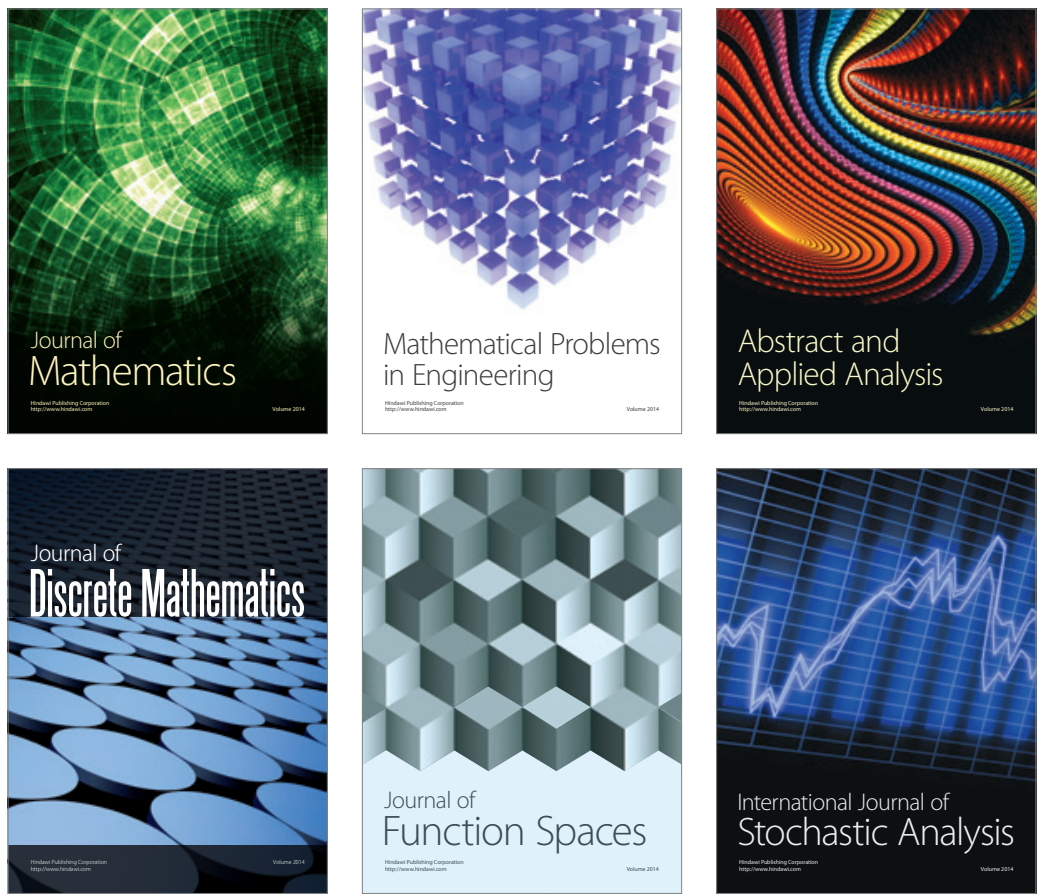

Journal of

Function Spaces

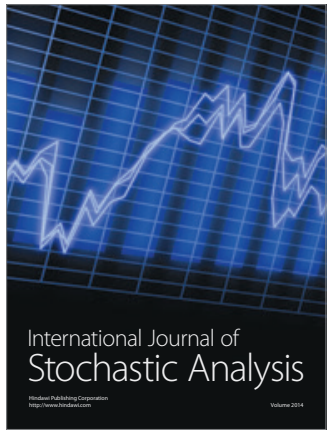

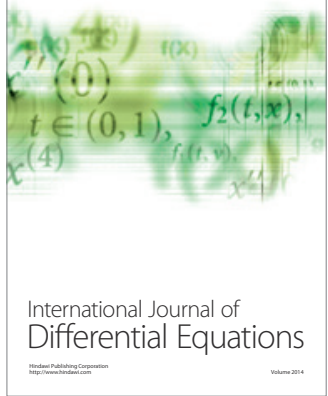
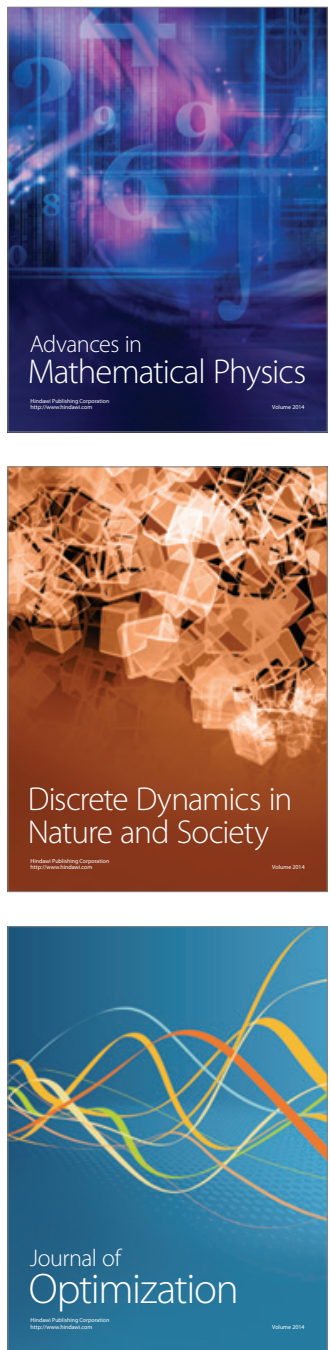